\title{
Borboletas (Papilionoidea e Hesperioidea) de Guarapuava e arredores, Paraná, Brasil: um inventário com base em 63 anos de registros
}

\author{
Diego Rodrigo Dolibaina ${ }^{1,2}$, Olaf Hermann Hendrik Mielke ${ }^{1}$ \& Mirna Martins Casagrande ${ }^{1}$ \\ ${ }^{1}$ Laboratório de Estudo de Lepidoptera Neotropical, Departamento de Zoologia, Universidade Federal \\ do Paraná - UFPR, CP 19020, CEP 81531-980, Curitiba, Paraná, Brasil \\ ${ }^{2}$ Autor para correspondência: Diego Rodrigo Dolibaina, e-mail: dirodrido@hotmail.com
}

DOLIBAINA, D.R., MIELKE, O.H.H. \& CASAGRANDE, M.M. Butterflies (Papilionoidea and Hesperioidea) from Guarapuava and vicinity, Paraná, Brazil: an inventory based on records of 63 years. Biota Neotrop. 11(1): http://www.biotaneotropica.org.br/v11n1/en/abstract?inventory+bn00211012011.

\begin{abstract}
Despite figuring among the countries with high diversity of butterflies, Brazil has little information available of its biodiversity. Paraná is one of brazilian states with the less known lepidopteran fauna. Only Curitiba and vicinity have a list of butterflies species published, while all other Paraná regions lack such information. Aiming to provide new informations about the butterflies fauna in Paraná state, this study compiles information of 63 years of collect (1944 to 2002 and from 2005 to 2010) in Guarapuava and vicinity, south-central Paraná, and emphasizes the typical grassland species, now nearly extinct in this region. Were recorded 689 species, 264 of these were recorded only in one of the sampling periods. About $6 \%$ are typical grassland species and fewer than half of those were recorded recently. Pieridae, Nymphalidae and Papilionidae latest records are more similar to earlier records than Riodinidae, Hesperiidae, and Lycaenidae. We suggest the creation of permanent conservation areas comprising grasslands, as they encompass a unique fauna and are threatened locally; furthermore, they are not protected by any conservation areas already established in the region.
\end{abstract}

Keywords: Diurnal Lepidoptera, richness, temporal variation, grassland, conservation.

DOLIBAINA, D.R., MIELKE, O.H.H. \& CASAGRANDE, M.M. Borboletas (Papilionoidea e Hesperioidea) de Guarapuava e arredores, Paraná, Brasil: um inventário com base em 63 anos de registros. Biota Neotrop. 11(1): http://www.biotaneotropica.org.br/v11n1/pt/abstract?inventory+bn00211012011.

Resumo: Apesar de estar entre os países com maior diversidade de borboletas, o Brasil possui pouca informação disponível que retrate essa biodiversidade. O Paraná é um dos estados brasileiros com a fauna de Lepidoptera menos conhecida. Apenas Curitiba e arredores possui uma lista de espécies robusta, enquanto as demais regiões paranaenses são desprovidas desse tipo de informação. Com o objetivo de aumentar o conhecimento sobre a fauna de borboletas do Paraná, o presente estudo fornece informações de 63 anos de coletas (1944 a 2002 e 2005 a 2010) em Guarapuava e arredores, região centro-sul paranaense, e indica as espécies características da fitofisionomia Campo Natural, hoje quase extinto nessa região. No total, 689 espécies foram coletadas, dessas 264 espécies estão representadas em apenas um dos períodos amostrais. Cerca de $6 \%$ da lista corresponde a espécies de Campo Natural e menos da metade foi encontrada recentemente. As famílias mais similares entre dados pretéritos e atuais foram Pieridae, Nymphalidae e Papilionidae, enquanto Riodinidae, Hesperiidae e Lycaenidae as mais dissimilares. Sugerimos a criação de unidades de conservação permanente que inclua a vegetação Campo Natural, visto que esta apresenta uma fauna peculiar e encontra-se ameaçada localmente, além de não ser protegida por unidades de conservação já estabelecidas na região.

Palavras-chave: Lepidoptera diurna, riqueza, variação temporal, campo natural, conservação. 


\section{Introdução}

Inventários de borboletas auxiliam na tomada de decisões com vistas à preservação (Brown Jr. \& Freitas 1999), além de subsidiar estudos de ecologia e fornecer fontes seguras de dados para estudos biogeográficos (Brown Jr. 1992). São ferramentas eficazes na produção da informação, subsidiando os programas de conservação (Carneiro et al. 2008b).

O Brasil é pouco representado por inventários de borboletas, e a maior parte de seu território está desprovido deste tipo de informação (Carneiro et al. 2008b).

Apesar de Carneiro et al. (2008b) indicarem as áreas da Caatinga e Amazônia ocidental como prioritárias para levantamentos de borboletas no Brasil, esses mesmos autores concluem que o estudo em qualquer localidade brasileira que objetive o conhecimento e inventário de espécies deve ser incentivado, visto a dimensão do país, sua grande biodiversidade, o acelerado ritmo de conversão dos ambientes naturais em áreas antropizadas e o baixo número de pesquisadores.

A Coleção Padre Jesus Santiago Moure da UFPR possui boa representação da fauna de borboletas do Paraná, entretanto, pouca informação foi publicada para o estado (Biezanko 1938a, b, Mielke, O.H.H. 1968, Mielke, C.G.C. 1995), e apenas Curitiba e arredores (Mielke, C.G.C. 1995) compreende uma amostragem robusta e focada em Papilionoidea e Hesperioidea.

A região de Guarapuava apresenta distintos componentes fitofisionômicos, apesar de amplamente fragmentada e isolada (Ribeiro et al. 2009) a Floresta Ombrófila Mista ou Floresta com Araucária está representada em todas as unidades de conservação da região, enquanto a fitofisionomia Estepe Gramíneo Lenhosa ou Campo Natural, que compreendia aproximadamente 171.000 ha da região (Maack 1968), não está presente nestas unidades de conservação, e o atual panorama aponta sua total conversão em áreas agropastoris.

Em 1944, quando os ambientes naturais de Guarapuava e região apresentavam bom estado de conservação, Hipólito Schneider, pesquisador autônomo de insetos, iniciou uma série de coletas, capturando principalmente Lepidoptera e Coleoptera por toda a região até o ano de 2002. Atualmente, parte de sua coleção está depositada na Universidade Estadual do Centro Oeste, no acervo do Museu de História Natural de Guarapuava, porém com caráter didático, enquanto outra parte encontra-se em sua residência.

Com o objetivo de aumentar o conhecimento disponível das borboletas no Estado do Paraná, o presente estudo fornece a lista de espécies de borboletas ocorrentes em Guarapuava e arredores, Paraná, Brasil, compilando dados pretéritos e atuais, além de indicar as espécies características de Campo Natural, um ambiente criticamente ameaçado nesta região.

\section{Materiais e Métodos}

A região de estudo é composta originalmente por três fitofisionomias, Floreta Ombrófila Mista, Campos Naturais e Floresta Estacional Semidecidual, sendo a última, encontrada apenas nas calhas dos Rios Ivaí e Iguaçu. As áreas florestais atualmente estão mais bem representadas, com $15,22 \%$ de sua área original (Sociedade... 1996). Já dos Campos Naturais, dominantes em tamanho, não se tem registros da pequena fração remanescente. No Paraná, esta vegetação distribuía-se pelos três planaltos, sendo que no terceiro, além da região de Guarapuava também ocorre no planalto de Palmas (Maack 1968) e em localidades adjacentes em Santa Catarina.

O clima na região é do tipo $\mathrm{Cfb}$, sem estação seca (Maack 1968). A temperatura média anual é $17,1^{\circ} \mathrm{C}$, ocorrendo pelo menos 10 geadas por ano. A precipitação média anual é de $1.923 \mathrm{~mm}$. Todos os dados meteorológicos correspondem ao período de 1976 a 2009 (Instituto... 2010). A altitude oscila entre 470 e 1.300 m.
Foram compilados dados de borboletas coletadas em Guarapuava e arredores, provenientes da coleção particular de Hipólito Schneider, do Museu de Ciências Naturais de Guarapuava - UNICENTRO e da Coleção Padre Jesus Santiago Moure - UFPR (DZUP), este último produto de coletas dos autores juniores. Esse banco de dados constitui as informações de espécies encontradas na região entre os anos de 1944 a 2002 (primeiro período amostral), totalizando 58 anos de registros. Como nesse período Guarapuava englobava inúmeros municípios hoje desmembrados (Pinhão, Inácio Martins, Candói, Cantagalo, Marquinho, Turvo, Reserva do Iguaçu, Campina do Simão, Goioxin), e os dados de etiqueta em sua maioria constam "Guarapuava", considerou-se que os exemplares poderiam ser provenientes de qualquer um dos atuais municípios acima citados. Algumas localidades especificadas em etiqueta são indicadas (Figura 1).

Entre abril de 2005 a março de 2010 (segundo período amostral), o primeiro autor iniciou suas coletas, que somadas compõem cinco anos de registros. Nesse caso, as mesmas foram decorrentes de coletas nos municípios de Turvo, Guarapuava e algumas poucas em Prudentópolis (Figura 1).

Borboletas foram coletadas entre 8:00 e 18:00 horas, ocasionalmente até o crepúsculo para coletar Brassolinae e Hesperiidae crepusculares (Mielke \& Casagrande 1998), através dos métodos usuais utilizados para Lepidoptera, ou seja, rede entomológica e armadilhas contendo iscas de banana fermentada para as espécies frugívoras. Posteriormente, os exemplares foram montados e etiquetados conforme normas internacionais de preparação. A identificação foi feita com base em exemplares pré-identificados na Coleção Padre Jesus Santiago Moure, além de revisões de gêneros disponíveis em literatura. A classificação e nomenclatura seguem Lamas (2004). Os exemplares coletados encontram-se depositados na Coleção Padre Jesus Santiago Moure - UFPR (DZUP), no Museu de História Natural de Guarapuava - UNICENTRO e na coleção particular de Hipólito Schneider.

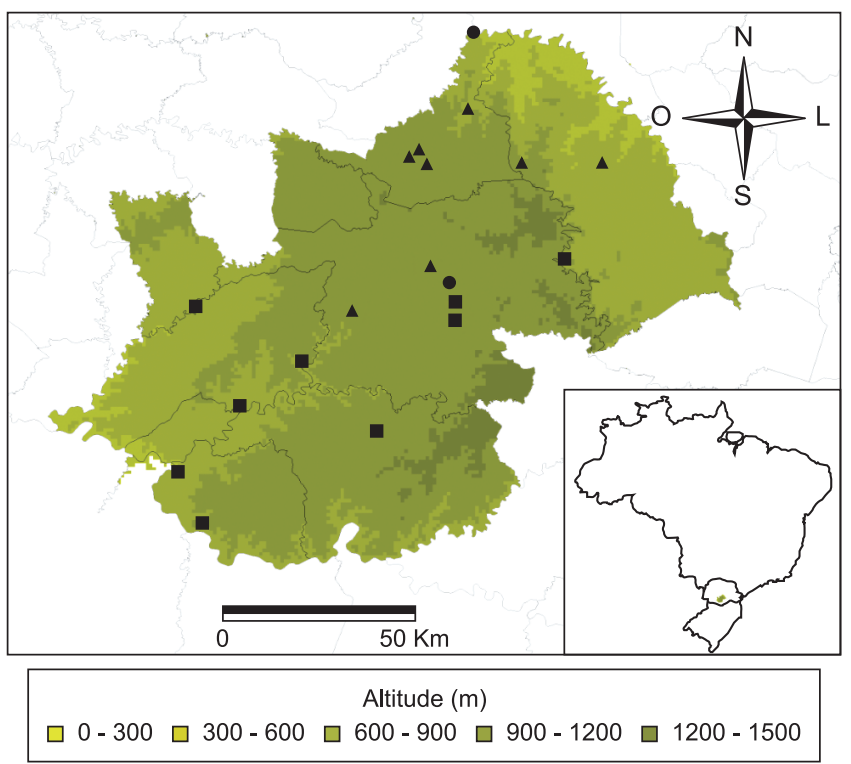

Figura 1. Pontos de coletas de borboletas na região de Guarapuava, Paraná, Brasil. Região de Guarapuava em destaque. Círculos = pontos de coletas coincidentes de Hipólito Schneider e Diego R. Dolibaina. Quadrados = pontos de coletas de Hipólito Schneider e/ou Olaf Mielke e Mirna Martins Casagrande. Triângulos $=$ pontos de coletas de Diego R. Dolibaina.

Figure 1. Collection points of butterflies at Guarapuava region, Paraná, Brazil. Guarapuava region highlighted. Circles $=$ coincident collections points of Hipólito Schneider and Diego R. Dolibaina. Squares = collections points of Hipólito Schneider and/or Olaf Mielke and Mirna Martins Casagrande. Triangles $=$ collections points of Diego R. Dolibaina 
Assim, a lista total de espécies é composta por dados correspondentes de 63 anos, e engloba grande parte da região denominada centro-sul paranaense ou planalto de Guarapuava (Maack 1968). Espécies com ocorrência presumível não foram consideradas, como em Emery et al. (2006) e Mielke et al. (2008).

Com o intuito de verificar a similaridade da riqueza encontrada entre dados pretéritos e atuais, utilizou-se o índice de similaridade de Sörensen (Is), com resultados apresentados em porcentagem.

Ainda na lista, indicam-se as espécies da região que são características de Campo Natural. Segundo Maack (1968, p. 26), Campo Natural ou por ele também chamado campos limpos (estepes de gramíneas baixas), caracteriza-se por: "[...] "extensas áreas de gramíneas baixas desprovidas de arbustos, ocorrendo matas ou capões limitados nas depressões em torno das nascentes"[...]", como nas figuras 97-102 e 204-206 de seu trabalho. Iserhard et al. (2010) foram os únicos a fornecerem a lista de borboletas amostradas nesta vegetação no Rio Grande do Sul. Considerou-se borboletas dessas áreas, aquelas coletadas estritamente nessa vegetação, tanto em campo seco quanto úmido. Eventualmente, estas podem ocorrer em Campos de Altitude da Serra do Mar, na Mantiqueira (Ebert 1969), no bioma Pampa do Rio Grande do Sul (Biezanko 1960, 1963, Biezanko \& Freitas 1938, Biezanko \& Mielke 1973, Biezanko et al. 1978, Link et al. 1977, Paz et al. 2008) e algumas poucas em Cerrado (Pinheiro \& Emery 2006, Mielke et al. 2008).

\section{Resultados}

Em 63 anos de coletas de borboletas do Planalto de Guarapuava, foram listadas 689 espécies representadas por 255 Hesperiidae (uma com duas subespécies), 19 Papilionidae (duas com duas subespécies), 33 Pieridae, 96 Lycaenidae, 76 Riodinidae e 210 Nymphalidae (Tabelas 1 e 2). Dessas, 574 foram registradas no primeiro e 543 no segundo período amostral (Tabelas 1 e 2). Recentemente (entre novembro/2009 a fevereiro/2010), acrescentou-se 20 novos registros à lista, entre Hesperiidae, Lycaenidae, Riodinidae e Nymphalidae, sugerindo que a riqueza regional seja maior que a atual.

Mesmo os dados pretéritos serem constituídos de apenas 31 espécies a mais que os atuais, apenas $61,8 \%$ das espécies são comuns entre as amostras, ou seja, 264 espécies de borboletas foram exclusivas a dados pretéritos (147 sp.) ou atuais (117 sp.) (Tabela 2). Hesperiidae (128 sp.), Riodinidae (39 sp.) e Lycaenidae (37 sp.) responderam por $79,1 \%$ das espécies exclusivas, portanto, metade dos Hesperiidae e Riodinidae são compostos por espécies amostradas apenas em um dos períodos amostrais, assim como 43,7\% dos Lycaenidae (Tabela 2). Ainda quanto às espécies exclusivas, 25 são características de Campo Natural, e 88,5\% destas ocorreram apenas no primeiro período amostral. Diferente das 237 espécies de mata, distribuídas igualmente entre os dados pretéritos e atuais.

A similaridae foi alta para Pieridae, Nymphalidae e Papilionidae, enquanto Riodinidae, Hesperiidae e Lycaenidae foram menos similares (Tabela 2). Apesar de 87,2\% das espécies de Nymphalidae serem similares entre as amostras, Limenitidinae, representada exclusivamente por espécies do gênero Adelpha, teve acentuada dissimilaridade, com apenas $42,1 \%$ das espécies correspondentes entre os períodos (Tabela 2).

As espécies de borboletas características de Campo Natural representam 6,1\% (42 sp.) da fauna total (Tabelas 1 e 2). Novamente Hesperiidae (26 sp.) foi a detentora de maior riqueza, seguida de Riodinidae (6 sp.), Nymphalidae (6 sp.), Lycaenidae (2 sp.), Papilionidae (1 sp.) e Pieridae (1 sp.) (Tabela 2). No primeiro período amostral foram registradas $38 \mathrm{sp}$ e no segundo 19 sp dessa fitofisionomia.
Algumas espécies que constam na lista da fauna ameaçada do Estado do Paraná (Mielke \& Casagrande 2004) foram registradas nesse estudo. São elas, Euryades corethrus (Boisduval, 1836), Pampasatyrus glaucope glaucope (C. \& R. Felder, 1867), Cyanophrys bertha (Jones, 1912) e Charonias theano (Boisduval, 1836), a última presente na recente versão da lista brasileira da fauna ameaçada de extinção (Casagrande \& Mielke 2008). As duas primeiras espécies são endêmicas de Campo Natural e apenas $E$. corethrus e $C$. bertha foram registradas no segundo período. Para maiores detalhes sobre novos dados de distribuição e registros dessas e outras borboletas ameaçadas do Paraná, veja Dolibaina et al. (2010).

Três espécies foram representadas por duas subespécies cada, duas em Papilionidae e uma em Hesperiidae. Enquanto as subespécies Parides bunichus bunichus (Hübner, [1821])) e P. b. perrhebus (Boisduval, 1836) ocorrem em altitudes distintas (acima de $800 \mathrm{~m}$ e a $500 \mathrm{~m}$, respectivamente) as subespécies Mimoides lysithous lysithous (Hübner, [1821]) e M. l. rurik (Eschscholtz, 1821) e Epargyreus socus socus Hübner, 1825 e E. s. pseudexadeus Westwood, 1852 foram registradas juntas.

\section{Discussão}

A região de Guarapuava possui elevada riqueza de borboletas, constituindo um dos sítios mais ricos no domínio da Floresta Ombrófila Mista (Biezanko 1938 a, b, Biezanko \& Pitoñ 1941, Mielke, C.G.C. 1995, Teston \& Corseuil 1999, 2000, 2002, Corseuil et al. 2004, Iserhard et al. 2010) e do Sul do Brasil (Paz et al. 2008, Giovenardi et al. 2008, Sackis \& Morais 2008, Carneiro et al. 2008a, Bonfantti et al. 2009) já documentado (ver Carneiro et al. 2008b para demais bibliografias). Este número é expressivo visto que a região apresenta inverno rigoroso devido à sua altitude (grande parte acima de $800 \mathrm{~m}$ ), e se localiza na porção subtropical do Brasil. A altitude média é de $900 \mathrm{~m}$, porém há locais em que esta atinge $470 \mathrm{~m}$, com a ocorrência da Floresta Estacional Semidecídual, contribuindo com uma fauna distinta. Segundo Brown Jr. \& Freitas (1999, 2000), para a localidade de Joinville, Santa Catarina (uma área de Floresta Ombrófila Densa de baixa altitude), constam 796 sp., no entanto, a lista jamais foi publicada.

A lista também apresenta riqueza similar a localidades de Floresta Estacional (Ebert 1969 (Poços de Caldas), Brown Jr. 1992 (Serra do Japi), Mielke \& Casagrande 1998 (Morro do Diabo), Brown Jr. \& Freitas 2000 (Campinas), Bustos 2009 (Parque Nacional del Iguazú - Argentina)), todos em zona tropical, exceto Bustos (2009). Apesar disso, tais listas retratam uma fauna bastante peculiar, não coincidindo as espécies pertencentes às famílias mais diversificadas como é o caso de Hesperiidae, Lycaenidae e Riodinidae com as do presente estudo.

Com base nos dados obtidos e na recente adição de 20 novos registros, sugere-se que a região possua mais espécies que as apresentadas na atual lista. Os esforços de coleta devem ser direcionados em locais não amostrados da região, especialmente em áreas de transição entre a Floresta Ombrófila Mista e a Floresta Estacional Semidecidual.

Diferenças na composição das espécies encontradas entre os dois períodos amostrais podem ser explicados pelo distinto esforço amostral (pois o primeiro período possui 53 anos a mais que o segundo), a alteração do ambiente natural (Uehara-Prado et al. 2007), especialmente os Campos Naturais, as coletas terem sido realizadas em locais não coincidentes entre as amostras (Summerville et al. 2003) (Figura 1), pela aptidão e experiência dos pesquisadores (Carneiro et al. 2008a) e pela possível nova ocupação de espécies antes não encontradas na região (Brown Jr. 1992).

Baixas similaridades encontradas para Hesperiidae, Lycaenidae e Riodinidae são atreladas à grande riqueza dessas famílias, seu pequeno tamanho e vôo rápido, levando consequentemente a dificuldades de amostragem, diferindo de Nymphalidae, Pieridae e 
Tabela 1. Lista das borboletas Hesperioidea e Papilionoidea de Guarapuava e arredores, Paraná, Brasil. * indica espécies de Campo Natural.

Table 1. List of Hesperiodea and Papilionoidea butterflies from Guarapuava and vicinity, Paraná, Brazil. * Indicates species from Grassland.

\begin{tabular}{|c|c|c|}
\hline \multirow[t]{2}{*}{ *Espécies/Subespécie } & 1947 & 2005 \\
\hline & 2002 & 2010 \\
\hline \multicolumn{3}{|l|}{ Hesperioidea - Hesperiidae } \\
\hline \multicolumn{3}{|l|}{ Pyrrhopyginae } \\
\hline \multicolumn{3}{|l|}{ Oxynetrini } \\
\hline Olafia roscius roscius (Hopffer, 1874) & $\mathrm{X}$ & $\mathrm{X}$ \\
\hline \multicolumn{3}{|l|}{ Passovini } \\
\hline Myscelus amystis epigona Herrich-Schäffer, 1869 & $\mathrm{X}$ & - \\
\hline \multicolumn{3}{|l|}{ Pyrrhopygini } \\
\hline Elbella adonis (Bell, 1931) & $\mathrm{X}$ & $\mathrm{X}$ \\
\hline Elbella mariae mariae (Bell, 1931) & $\mathrm{X}$ & $\mathrm{X}$ \\
\hline Pseudocroniades machaon machaon (Westwood, 1852) & $\mathrm{X}$ & $\mathrm{X}$ \\
\hline Pyrrhopyge charybdis charybdis Westwood, 1852 & $\mathrm{X}$ & $\mathrm{X}$ \\
\hline Sarbia antias (C. Felder \& R. Felder, 1859) & $\mathrm{X}$ & - \\
\hline Sarbia curitiba Mielke \& Casagrande, 2002 & $\mathrm{X}$ & $\mathrm{X}$ \\
\hline Sarbia damippe Mabille \& Boullet, 1908 & $\mathrm{X}$ & $\mathrm{X}$ \\
\hline Sarbia pertyi (Plötz, 1879) & $\mathrm{X}$ & $\mathrm{X}$ \\
\hline Sarbia xanthippe spixii (Plötz, 1879) & $\mathrm{X}$ & $\mathrm{X}$ \\
\hline \multicolumn{3}{|l|}{ Pyrginae } \\
\hline \multicolumn{3}{|l|}{ Eudamini } \\
\hline Aguna asander asander (Hewitson, 1867) & $\mathrm{X}$ & $\mathrm{X}$ \\
\hline Aguna glaphyrus (Mabille, 1888) & $\mathrm{X}$ & - \\
\hline Astraptes alardus alardus (Stoll, 1790) & $\mathrm{X}$ & - \\
\hline Astraptes anaphus anaphus (Cramer, 1777) & $\mathrm{X}$ & - \\
\hline Astraptes aulus (Plötz, 1881) & $\mathrm{X}$ & $\mathrm{X}$ \\
\hline Astraptes cretatus adoba Evans, 1952 & - & $\mathrm{X}$ \\
\hline Astraptes creteus siges (Mabille, 1903) & $\mathrm{X}$ & - \\
\hline Astraptes elorus (Hewitson, 1867) & $\mathrm{X}$ & $\mathrm{X}$ \\
\hline Astraptes erycina (Plötz, 1881) & $\mathrm{X}$ & - \\
\hline Astraptes fulgerator fulgerator (Walch, 1775) & $\mathrm{X}$ & $\mathrm{X}$ \\
\hline Astraptes fulgor (Hayward, 1939) & $\mathrm{X}$ & - \\
\hline Astraptes naxos (Hewitson, 1867) & $\mathrm{X}$ & $\mathrm{X}$ \\
\hline Autochton integrifascia (Mabille, 1891) & $\mathrm{X}$ & $\mathrm{X}$ \\
\hline Autochton neis (Geyer, 1832) & - & $\mathrm{X}$ \\
\hline Autochton zarex (Hübner, 1818) & - & $\mathrm{X}$ \\
\hline Celaenorrhinus eligius punctiger (Burmeister, 1878) & $\mathrm{X}$ & - \\
\hline Celaenorrhinus similis Hayward, 1933 & - & $\mathrm{X}$ \\
\hline Chioides catillus catillus (Cramer, 1779) & $\mathrm{X}$ & $\mathrm{X}$ \\
\hline Codatractus aminias (Hewitson, 1867) & $\mathrm{X}$ & $\mathrm{X}$ \\
\hline Dyscophellus ramusis damias (Plötz, 1882) & $\mathrm{X}$ & - \\
\hline Epargyreus exadeus exadeus (Cramer, 1779) & $\mathrm{X}$ & - \\
\hline Epargyreus socus socus Hübner, [1825] & $\mathrm{X}$ & - \\
\hline Epargyreus socus pseudexadeus Westwood, 1852 & $\mathrm{X}$ & $\mathrm{X}$ \\
\hline Phanus australis L.D. Miller, 1965 & $\mathrm{X}$ & $\mathrm{X}$ \\
\hline Phocides charon (C. Felder \& R. Felder, 1859) & $\mathrm{X}$ & $\mathrm{X}$ \\
\hline Phocides pialia pialia (Hewitson, 1857) & $\mathrm{X}$ & $\mathrm{X}$ \\
\hline Phocides polybius phanias (Burmeister, 1880) & $\mathrm{X}$ & - \\
\hline Polygonus leo leo (Gmelin, [1790]) & $\mathrm{X}$ & $\mathrm{X}$ \\
\hline Polygonus savigny savigny (Latreille, [1824]) & $\mathrm{X}$ & $\mathrm{X}$ \\
\hline Proteides mercurius mercurius (Fabricius, 1787) & $\mathrm{X}$ & $\mathrm{X}$ \\
\hline Urbanus albimargo rica Evans, 1952 & $\mathrm{X}$ & $\mathrm{X}$ \\
\hline Urbanus chalco (Hübner, 1823) & $\mathrm{X}$ & $\mathrm{X}$ \\
\hline Urbanus dorantes dorantes (Stoll, 1790) & $\mathrm{X}$ & $\mathrm{X}$ \\
\hline
\end{tabular}

\begin{tabular}{lcc}
\hline *Espécies/Subespécie & $\mathbf{1 9 4 7}$ & $\mathbf{2 0 0 5}$ \\
\cline { 2 - 3 } & $\mathbf{2 0 0 2}$ & $\mathbf{2 0 1 0}$ \\
\hline Urbanus esta Evans, 1952 & $\mathrm{X}$ & $\mathrm{X}$ \\
Urbanus procne (Plötz, 1880) & $\mathrm{X}$ & $\mathrm{X}$ \\
Urbanus proteus proteus (Linnaeus, 1758) & $\mathrm{X}$ & $\mathrm{X}$ \\
Urbanus simplicius (Stoll, 1790) & - & $\mathrm{X}$ \\
Urbanus pronta Evans, 1952 & - & $\mathrm{X}$ \\
Urbanus teleus (Hübner, 1821) & $\mathrm{X}$ & $\mathrm{X}$ \\
*Urbanus zagorus (Plötz, 1880) & $\mathrm{X}$ & -
\end{tabular}

Pyrgini

Achlyodes busirus rioja Evans, 1953

Achlyodes mithridates thraso (Hübner, [1807])

Aethilla echina coracina Butler, 1870

Anastrus sempiternus simplicior (Möschler, 1877)

Anisochoria pedaliodina extincta Hayward, 1933

Anisochoria sublimbata Mabille, 1883

Anisochoria subpicta Schaus, 1902

Antigonus erosus (Hübner, [1812])

Antigonus liborius areta Evans, 1953

Antigonus minor Mielke, 1980

Carrhenes canescens pallida Röber, 1925

Chiomara asychis autander (Mabille, 1891)

*Cogia calchas (Herrich-Schäffer, 1869)

*Cogia cerradicola (Mielke, 1967)

Cycloglypha stellita Zikán, 1938

Cycloglypha thrasibulus thrasibulus (Fabricius, 1793)

Ebrietas anacreon anacreon (Staudinger, 1876)

Erynnis funeralis (Scudder \& Burgess, 1870)

*Gesta austerus (Schaus, 1902)

Gesta gesta (Herrich-Schäffer, 1869)

Gorgythion begga begga (Prittwitz, 1868)

Grais stigmaticus stigmaticus (Mabille, 1883)

Heliopetes laviana laviana (Hewitson, 1868)

Helioptes leucola (Hewitson, 1868)

Heliopetes libra Evans, 1944

Heliopetes ochroleuca J. Zikán, 1938

Heliopetes omrina (Butler, 1870)

Milanion leucaspis (Mabille, 1878)

Mylon maimon (Fabricius, 1775)

Nisoniades bipuncta (Schaus, 1902)

Nisoniades castolus (Hewitson, 1878)

Nisoniades maura (Mabille \& Boullet, 1917)

Noctuana diurna (Butler, 1870)

Ocella monophthalma (Plötz, 1884)

Oechydrus chersis evelinda (Butler, 1870)

Ouleus fridericus riona Evans, 1953

Polyctor polyctor polyctor (Prittwitz, 1868)

Pyrgus orcus (Stoll, 1780)

Pyrgus orcynoides (Giacomelli, 1928)

Pythonides lancea (Hewitson, 1868)

Quadrus u-lucida mimus (Mabille \& Boullet, 1917)

Sostrata bifasciata bifasciata (Ménétriés, 1829)

X $\quad \mathrm{X}$

$X \quad X$

$\mathrm{X}-$

$-\quad X$

$\mathrm{X}-$

X $\quad X$

$-\quad X$

$\mathrm{X} \quad-$

$X \quad X$

$\mathrm{X}-$

$\mathrm{X} \quad \mathrm{X}$

$\mathrm{X}-$

$X \quad X$

$\mathrm{X}-$

$-\quad X$

$-\quad X$

$\begin{array}{ll}X & X\end{array}$

$\mathrm{X} \quad \mathrm{X}$

$\mathrm{X}-$

X $\mathrm{X}$

X $\quad X$

- $\quad X$

X -

X $\mathrm{X}$

X X

- X

X X

X X

- X

X X

- X

X -

X $\mathrm{X}$

- $\quad X$

X X

- $\quad X$

X X

X X

X X

X X

$-\quad X$

Staphylus ascalon (Staudinger, 1876)

X X

$-\quad X$


Tabela 1. Continuação...

\begin{tabular}{|c|c|c|}
\hline \multirow[t]{2}{*}{ *Espécies/Subespécie } & \multirow{2}{*}{$\begin{array}{l}1947 \\
2002 \\
\end{array}$} & \multirow{2}{*}{$\begin{array}{ll}2005 \\
2010\end{array}$} \\
\hline & & \\
\hline Staphylus coecatus (Mabille, 1891) & - & $\mathrm{X}$ \\
\hline Staphylus incisus (Mabille, 1878) & $\mathrm{X}$ & $\mathrm{X}$ \\
\hline Staphylus minor minor Schaus, 1902 & $\mathrm{X}$ & - \\
\hline Staphylus musculus (Burmeister, 1875) & $\mathrm{X}$ & - \\
\hline Telemiades amphion marpesus (Hewitson, 1876) & - & $\mathrm{X}$ \\
\hline Theagenes dichrous (Mabille, 1878) & $\mathrm{X}$ & $\mathrm{X}$ \\
\hline Trina geometrina geometrina $($ C. Felder \& R. Felder, 1867) & $\mathrm{X}$ & $\mathrm{X}$ \\
\hline *Viola alicus (Schaus, 1902) & $\mathrm{X}$ & - \\
\hline *Viola minor (Hayward, 1933) & $\mathrm{X}$ & $\mathrm{X}$ \\
\hline Xenophanes tryxus (Stoll, 1780) & $\mathrm{X}$ & $\mathrm{X}$ \\
\hline Zera hyacinthinus servius (Plötz, 1884) & - & $\mathrm{X}$ \\
\hline Zera tetrastigma erisichthon (Plötz, 1884) & $\mathrm{X}$ & $\mathrm{X}$ \\
\hline *Zopyrion evenor evenor Godman, 1901 & $\mathrm{X}$ & - \\
\hline \multicolumn{3}{|l|}{ Hesperiinae } \\
\hline Aides duma argyrina Cowan, 1970 & $\mathrm{X}$ & - \\
\hline Alera furcata Mabille, 1891 & $\mathrm{X}$ & - \\
\hline Alera metallica (Riley, 1921) & - & $\mathrm{X}$ \\
\hline Anatrytone perfida (Möschler, 1879) & - & $\mathrm{X}$ \\
\hline *Ancyloxypha nitedula (Burmeister, 1878) & $\mathrm{X}$ & - \\
\hline Anthoptus epictetus (Fabricius, 1793) & $\mathrm{X}$ & $\mathrm{X}$ \\
\hline Arotis derasa brunnea (Mielke, 1972) & $\mathrm{X}$ & $\mathrm{X}$ \\
\hline *Artines satyr Evans, 1955 & - & $\mathrm{X}$ \\
\hline Callimormus interpunctata (Plötz, 1884) & $\mathrm{X}$ & $\mathrm{X}$ \\
\hline Callimormus rivera (Plötz, 1882) & $\mathrm{X}$ & $\mathrm{X}$ \\
\hline Calpodes ethlius (Stoll, 1782) & - & $\mathrm{X}$ \\
\hline Carystus phorcus claudianus (Latreille, [1824]) & $\mathrm{X}$ & - \\
\hline Chalcone santarus (Bell, 1940) & - & $\mathrm{X}$ \\
\hline Cobalopsis hazarma (Hewitson, 1877) & $\mathrm{X}$ & - \\
\hline Cobalopsis catocala (Herrich-Schäffer, 1869) & $\mathrm{X}$ & - \\
\hline Cobalopsis miaba (Schaus, 1902) & $\mathrm{X}$ & $\mathrm{X}$ \\
\hline Cobalopsis nero (Herrich-Schäffer, 1869) & $\mathrm{X}$ & - \\
\hline Cobalopsis obscurior (Hayward, 1934) & $\mathrm{X}$ & - \\
\hline Cobalopsis vorgia (Schaus, 1902) & $\mathrm{X}$ & - \\
\hline Cobalopsis sp. & $\mathrm{X}$ & - \\
\hline Conga chydaea (Butler, 1877) & $\mathrm{X}$ & $\mathrm{X}$ \\
\hline Conga iheringii (Mabille, 1891) & $\mathrm{X}$ & $\mathrm{X}$ \\
\hline Conga immaculata (Bell, 1930) & $\mathrm{X}$ & $\mathrm{X}$ \\
\hline *Conga urqua (Schaus, 1902) & $\mathrm{X}$ & $\mathrm{X}$ \\
\hline *Conga zela (Plötz, 1883) & $\mathrm{X}$ & - \\
\hline *Copaeodes jean favor Evans, 1955 & $\mathrm{X}$ & $\mathrm{X}$ \\
\hline Corticea corticea (Plötz, 1882) & $\mathrm{X}$ & $\mathrm{X}$ \\
\hline *Corticea immocerinus (Hayward, 1934) & $\mathrm{X}$ & $\mathrm{X}$ \\
\hline Corticea lysias potex Evans, 1955 & $\mathrm{X}$ & - \\
\hline Corticea obscura Mielke, 1969 & $\mathrm{X}$ & $\mathrm{X}$ \\
\hline Corticea sp. n. & $\mathrm{X}$ & $\mathrm{X}$ \\
\hline Cumbre cumbre (Schaus, 1902) & - & $\mathrm{X}$ \\
\hline Cumbre sp. n. & - & $\mathrm{X}$ \\
\hline Cumbre sp. n. & - & $\mathrm{X}$ \\
\hline Cymaenes cavalla Evans, 1955 & $\mathrm{X}$ & $\mathrm{X}$ \\
\hline Cymaenes distigma (Plötz, 1882) & $\mathrm{X}$ & $\mathrm{X}$ \\
\hline Cymaenes gisca Evans, 1955 & $\mathrm{X}$ & - \\
\hline Cymaenes laurelolus loxa Evans, 1955 & - & $\mathrm{X}$ \\
\hline
\end{tabular}

\begin{tabular}{|c|c|c|}
\hline \multirow[t]{2}{*}{ *Espécies/Subespécie } & 1947 & 2005 \\
\hline & 2002 & 2010 \\
\hline Cymaenes lepta (Hayward, 1939) & - & $\mathrm{X}$ \\
\hline Cymaenes odilia odilia (Burmeister, 1878) & $\mathrm{X}$ & $\mathrm{X}$ \\
\hline Cymaenes perloides (Plötz, 1882) & - & $\mathrm{X}$ \\
\hline Cymaenes tripunctata tripunctata (Latreille, [1824]) & $\mathrm{X}$ & $\mathrm{X}$ \\
\hline Cynea popla Evans, 1955 & - & $\mathrm{X}$ \\
\hline Decinea dama (Herrich-Schäffer, 1869) & $\mathrm{X}$ & - \\
\hline Decinea decinea decinea (Hewitson, 1876) & - & $\mathrm{X}$ \\
\hline Decinea percosius (Godman, 1900) & $\mathrm{X}$ & - \\
\hline *Euphyes cherra Evans, 1955 & $\mathrm{X}$ & $\mathrm{X}$ \\
\hline Euphyes subferrugineus biezankoi Mielke, 1972 & $\mathrm{X}$ & $\mathrm{X}$ \\
\hline Eutychide physcella (Hewitson, 1866) & $\mathrm{X}$ & $\mathrm{X}$ \\
\hline Evansiella cordela (Plötz, 1882) & $\mathrm{X}$ & $\mathrm{X}$ \\
\hline Gallio carasta (Schaus, 1902) & - & $\mathrm{X}$ \\
\hline Hansa hyboma (Plötz, 1886) & $\mathrm{X}$ & - \\
\hline Hylephila ancora (Plötz, 1883) & $\mathrm{X}$ & - \\
\hline Hylephila phyleus phyleus (Drury, 1773) & $\mathrm{X}$ & $\mathrm{X}$ \\
\hline Justinia kora (Hewitson, 1877) & $\mathrm{X}$ & $\mathrm{X}$ \\
\hline Lamponia lamponia (Hewitson, 1876) & - & $\mathrm{X}$ \\
\hline Lento krexoides (Hayward, 1940) & - & $\mathrm{X}$ \\
\hline Lerema duroca lenta Evans, 1955 & $\mathrm{X}$ & $\mathrm{X}$ \\
\hline Lerodea eufala eufala (W.H. Edwards, 1869) & $\mathrm{X}$ & $\mathrm{X}$ \\
\hline Levina levina (Plötz, 1884) & $\mathrm{X}$ & - \\
\hline Libra anatolica (Plötz, 1883) & $\mathrm{X}$ & - \\
\hline Lucida lucia lucia (Capronnier, 1874) & $\mathrm{X}$ & $\mathrm{X}$ \\
\hline Lucida ranesus (Schaus, 1902) & $\mathrm{X}$ & $\mathrm{X}$ \\
\hline Lucida sp. n. & - & $\mathrm{X}$ \\
\hline Lycas argentea (Hewitson, 1866) & $\mathrm{X}$ & $\mathrm{X}$ \\
\hline Lychnuchoides ozias ozias (Hewitson, 1878) & $\mathrm{X}$ & $\mathrm{X}$ \\
\hline Lychnuchus celsus (Fabricius, 1793) & $\mathrm{X}$ & - \\
\hline Metron chrysogastra hypodesma (Plötz, 1882) & $\mathrm{X}$ & - \\
\hline Metron oropa (Hewitson, 1877) & $\mathrm{X}$ & $\mathrm{X}$ \\
\hline Miltomiges cinnamomea (Herrich-Schäffer, 1869) & - & $\mathrm{X}$ \\
\hline Mnasitheus nella Evans, 1955 & $\mathrm{X}$ & - \\
\hline Mnasitheus ritans (Schaus, 1902) & - & $\mathrm{X}$ \\
\hline Moeris striga striga (Geyer, 1832) & $\mathrm{X}$ & $\mathrm{X}$ \\
\hline *Molla molla Evans, 1955 & $\mathrm{X}$ & $\mathrm{X}$ \\
\hline Monca branca Evans, 1955 & $\mathrm{X}$ & - \\
\hline Nastra lurida (Herrich-Schäffer, 1869) & $\mathrm{X}$ & $\mathrm{X}$ \\
\hline Niconiades caeso (Mabille, 1891) & $\mathrm{X}$ & $\mathrm{X}$ \\
\hline Niconiades merenda (Mabille, 1878) & $\mathrm{X}$ & $\mathrm{X}$ \\
\hline Niconiades nikko Hayward, 1948 & $\mathrm{X}$ & - \\
\hline Nyctelius nyctelius nyctelius (Latreille, [1824]) & $\mathrm{X}$ & $\mathrm{X}$ \\
\hline Nyctelius paranensis (Schaus, 1902) & $\mathrm{X}$ & $\mathrm{X}$ \\
\hline Onophas columbaria distigma Bell, 1930 & $\mathrm{X}$ & - \\
\hline Onophas watsoni Bell, 1930 & - & $\mathrm{X}$ \\
\hline Orses cynisca (Swainson, 1821) & - & $\mathrm{X}$ \\
\hline Orses itea (Swainson, 1821) & $\mathrm{X}$ & $\mathrm{X}$ \\
\hline Orthos orthos hyalinus (Bell, 1930) & $\mathrm{X}$ & - \\
\hline Oxynthes corusca (Herrich-Schäffer, 1869) & - & $\mathrm{X}$ \\
\hline Panoquina lucas lucas (Fabricius, 1793) & $\mathrm{X}$ & $\mathrm{X}$ \\
\hline Papias phainis Godman, 1900 & $\mathrm{X}$ & - \\
\hline *Papias sp. n. & $\mathrm{X}$ & - \\
\hline
\end{tabular}


Tabela 1. Continuação...

\begin{tabular}{|c|c|c|}
\hline \multirow[t]{2}{*}{ *Espécies/Subespécie } & 1947 & 2005 \\
\hline & 2002 & 2010 \\
\hline Paracarystus evansi Hayward, 1938 & $\mathrm{X}$ & $\mathrm{X}$ \\
\hline Parphorus pseudecorus (Hayward, 1934) & - & $\mathrm{X}$ \\
\hline Perichares philetes adela (Hewitson, 1867) & $\mathrm{X}$ & $\mathrm{X}$ \\
\hline Perichares seneca seneca (Latreille, [1824]) & $\mathrm{X}$ & $\mathrm{X}$ \\
\hline Pheraeus argynnis (Plötz, 1884) & $\mathrm{X}$ & - \\
\hline *Polites vibex catilina (Plötz, 1886) & $\mathrm{X}$ & $\mathrm{X}$ \\
\hline *Pompeius dares (Plötz, 1883) & $\mathrm{X}$ & - \\
\hline Pompeius pompeius (Latreille, [1824]) & - & $\mathrm{X}$ \\
\hline Psoralis stacara (Schaus, 1902) & $\mathrm{X}$ & $\mathrm{X}$ \\
\hline Pyrrhopygopsis socrates socrates (Ménétriés, 1855) & $\mathrm{X}$ & $\mathrm{X}$ \\
\hline Quinta cannae (Herrich-Schäffer, 1869) & $\mathrm{X}$ & - \\
\hline Remella remus (Fabricius, 1798) & $\mathrm{X}$ & $\mathrm{X}$ \\
\hline Saliana longirostris (Sepp, [1840]) & $\mathrm{X}$ & - \\
\hline Saniba sabina (Plötz, 1882) & - & $\mathrm{X}$ \\
\hline Saturnus reticulata conspicuus (Bell, 1941) & $\mathrm{X}$ & $\mathrm{X}$ \\
\hline Sodalia coler (Schaus, 1902) & $\mathrm{X}$ & $\mathrm{X}$ \\
\hline Sucova sucova (Schaus, 1902) & - & $\mathrm{X}$ \\
\hline *Synale hylaspes (Stoll, 1781) & $\mathrm{X}$ & - \\
\hline Synapte malitiosa antistia (Plötz, 1882) & - & $\mathrm{X}$ \\
\hline Synapte silius (Latreille, [1824])) & $\mathrm{X}$ & - \\
\hline Thargella evansi Biezanko \& Mielke, 1973 & - & $\mathrm{X}$ \\
\hline Thespieus castor Hayward, 1948 & $\mathrm{X}$ & $\mathrm{X}$ \\
\hline Thespieus dalman (Latreille, [1824]) & $\mathrm{X}$ & $\mathrm{X}$ \\
\hline Thespieus ethemides (Burmeister, 1878) & $\mathrm{X}$ & $\mathrm{X}$ \\
\hline Thespieus jora Evans, 1955 & $\mathrm{X}$ & $\mathrm{X}$ \\
\hline Thespieus lutetia (Hewitson, 1866) & $\mathrm{X}$ & - \\
\hline Thespieus vividus (Mabille, 1891) & $\mathrm{X}$ & $\mathrm{X}$ \\
\hline Thespieus xarina Hayward, 1948 & $\mathrm{X}$ & $\mathrm{X}$ \\
\hline Thespieus xarippe xarippe (Butler, 1870) & $\mathrm{X}$ & $\mathrm{X}$ \\
\hline Thracides cleanthes cleanthes (Latreille, [1824]) & $\mathrm{X}$ & $\mathrm{X}$ \\
\hline Tisias lesueur lesueur (Latreille, [1824]) & $\mathrm{X}$ & - \\
\hline Tisias quadrata quadrata (Herrich-Schäffer, 1869) & $\mathrm{X}$ & - \\
\hline Vacerra caniola elva Evans, 1955 & - & $\mathrm{X}$ \\
\hline *Vehilius celeus vetus Mielke, 1969 & - & $\mathrm{X}$ \\
\hline Vehilius clavicula (Plötz, 1884) & $\mathrm{X}$ & $\mathrm{X}$ \\
\hline *Vehilius inca (Scudder, 1872) & $\mathrm{X}$ & - \\
\hline Vehilius stictomenes stictomenes (Butler, 1877) & $\mathrm{X}$ & $\mathrm{X}$ \\
\hline Vettius artona (Hewitson, 1868) & $\mathrm{X}$ & $\mathrm{X}$ \\
\hline Vettius diana diana (Plötz, 1886) & - & $\mathrm{X}$ \\
\hline Vettius diversa diversa (Herrich-Schäffer, 1869) & $\mathrm{X}$ & $\mathrm{X}$ \\
\hline Vettius fuldai (Bell, 1930) & $\mathrm{X}$ & - \\
\hline Vettius phyllus prona Evans, 1955 & - & $\mathrm{X}$ \\
\hline *Vidius mictra Evans, 1955 & - & $\mathrm{X}$ \\
\hline *Vidius nappa Evans 1955 & $\mathrm{X}$ & - \\
\hline *Vidius similis Mielke, 1980 & $\mathrm{X}$ & - \\
\hline *Vidius vidius (Mabille, 1891) & $\mathrm{X}$ & - \\
\hline Vinius letis (Plötz, 1883) & $\mathrm{X}$ & $\mathrm{X}$ \\
\hline Vinius pulcherrimus Hayward, 1834 & - & $\mathrm{X}$ \\
\hline Virga austrinus (Hayward, 1934) & $\mathrm{X}$ & $\mathrm{X}$ \\
\hline *Virga riparia Mielke, 1969 & $\mathrm{X}$ & - \\
\hline Wallengrenia premnas (Wallengren, 1860) & $\mathrm{X}$ & $\mathrm{X}$ \\
\hline Xeniades chalestra corna Evans, 1955 & $\mathrm{X}$ & - \\
\hline
\end{tabular}

\begin{tabular}{lcc}
\hline *Espécies/Subespécie & $\mathbf{1 9 4 7}$ & $\mathbf{2 0 0 5}$ \\
\cline { 2 - 4 } & $\mathbf{2 0 0 2}$ & $\mathbf{2 0 1 0}$ \\
\hline Xeniades orchamus orchamus (Cramer, 1777) & - & $\mathrm{X}$ \\
Xeniades victoria Evans, 1955 & - & $\mathrm{X}$ \\
Zenis minos (Latreille, [1824]) & $\mathrm{X}$ & $\mathrm{X}$ \\
Zenis jebus jebus (Plötz, 1882) & $\mathrm{X}$ & $\mathrm{X}$ \\
Heteropterinae & & \\
Dalla jelskyi aurosa J. Zikán, 1938 & $\mathrm{X}$ & - \\
Dardarina aspila Mielke, 1966 & $\mathrm{X}$ & $\mathrm{X}$ \\
Dardarina rana Evans, 1955 & $\mathrm{X}$ & -
\end{tabular}

Papilionoidea - Papilionidae

Papilioninae

Leptocircini

Mimoides lysithous lysithous (Hübner, [1821]) $\quad$ X $\quad$ X

Mimoides lysithous rurik (Eschscholtz, 1821) X -

Protesilaus helios (Rothschild \& Jordan, 1906) X X

Protesilaus protesilaus nigricornis (Staudinger, 1884) X -

Protesilaus stenodesmus (Rothschild \& Jordan, 1906) X -

Protesilaus telesilaus vitellus (Fruhstorfer, 1907) X -

Troidini

Battus polydamas polydamas (Linnaeus, 1758) $\quad$ X $\quad$ X

Battus polystictus polystictus (Butler, 1874) $\quad$ X $\quad \mathrm{X}$

*Euryades corethrus (Boisduval, 1836) $\quad$ X $\quad$ X

Parides agavus (Drury, 1782) $\quad$ X $\quad$ X

Parides anchises nephalion (Godart, 1819) $\quad$ X $\quad$ X

Parides bunichus bunichus (Hüner, [1821]) X X

Parides bunichus perhebus (Boiduval, 1836) X -

Parides proneus (Hübner, [1831]) X X

Papilionini

Heraclides anchisiades capys (Hübner, [1809]) X X

Heraclides androgeus androgeus (Cramer, 1775) X -

Heraclides astyalus astyalus (Godart, 1819) $\quad$ X X

Heraclides hectorides (Esper, 1794) X X

Heraclides thoas brasiliensis (Rothschild \& Jordan, 1906) X X X

Pterourus menatius cleotas (Gray, 1832) $\quad$ X $\quad$ X

Pterourus scamander scamander (Boisduval, 1836) $\quad$ X $\quad$ X

Pieridae

Dismorphiinae

Dismorphia amphinome astynome (Dalman, 1823) $\quad$ X $\quad$ X

Dismorphia astyocha Hübner, [1831] $\quad$ X $\quad$ X

Dismorphia melia (Godart, [1824]) X -

Dismorphia thermesia thermesia (Godart, 1819) $\quad$ X $\quad$ X

Enantia clarissa (Weymer, 1895) X X X

Enantia lina psamathe (Fabricius, 1793) $\quad \mathrm{X} \quad \mathrm{X}$

Pseudopieris nehemia nehemia (Boisduval, 1836) X X

Coliadinae

Aphrissa statira statira (Cramer, 1777) $\quad$ X $\quad$ X

Colias lesbia lesbia (Fabricius, 1775) $\quad$ X $\quad$ X

Eurema albula albula (Cramer, 1775) $\quad$ X $\quad$ X

Eurema arbela arbela Geyer, $1832 \quad$ X X

Eurema deva deva (Doubleday, 1847) X X

Eurema elathea flavescens (Chavannes, 1850) X X

*Eurema phiale paula (Röber, 1909) X X

Phoebis argante argante (Fabricius, 1775) $\quad \mathrm{X} \quad \mathrm{X}$ 
Tabela 1. Continuação...

\begin{tabular}{|c|c|c|}
\hline \multirow[t]{2}{*}{ *Espécies/Subespécie } & 1947 & 2005 \\
\hline & 2002 & 2010 \\
\hline Phoebis neocypris neocypris (Hübner, [1823]) & $\mathrm{X}$ & $\mathrm{X}$ \\
\hline Phoebis philea philea (Linnaeus, 1763) & $\mathrm{X}$ & $\mathrm{X}$ \\
\hline Phoebis sennae marcellina (Cramer, 1777) & $\mathrm{X}$ & $\mathrm{X}$ \\
\hline Pyrisitia leuce leuce (Boisduval, 1836) & $\mathrm{X}$ & $\mathrm{X}$ \\
\hline Pyrisitia nise tenella (Boisduval, 1836) & $\mathrm{X}$ & - \\
\hline Pyrisitia venusta venusta (Boisduval, 1836) & $\mathrm{X}$ & - \\
\hline Rhabdodryas trite banksi (Breyer, 1939) & $\mathrm{X}$ & $\mathrm{X}$ \\
\hline \multicolumn{3}{|l|}{ Pierinae } \\
\hline \multicolumn{3}{|l|}{ Anthocharidini } \\
\hline Hesperocharis erota (Lucas, 1852) & $\mathrm{X}$ & $\mathrm{X}$ \\
\hline Hesperocharis paranensis paranensis Schaus, 1898 & $\mathrm{X}$ & $\mathrm{X}$ \\
\hline \multicolumn{3}{|l|}{ Pierini } \\
\hline Ascia monuste orseis (Godart, 1819) & $\mathrm{X}$ & $\mathrm{X}$ \\
\hline Catasticta bithys (Hübner, [1831]) & $\mathrm{X}$ & $\mathrm{X}$ \\
\hline Charonias theano (Boisduval, 1836) & $\mathrm{X}$ & - \\
\hline Glutophrissa drusilla drusilla (Cramer, 1777) & $\mathrm{X}$ & $\mathrm{X}$ \\
\hline Leptophobia aripa balidia (Boisduval, 1836) & $\mathrm{X}$ & $\mathrm{X}$ \\
\hline Melete lycimnia petronia Fruhstorfer, 1907 & - & $\mathrm{X}$ \\
\hline Pereute swainsoni (Gray, 1832) & $\mathrm{X}$ & $\mathrm{X}$ \\
\hline Pieriballia viardi molione (Fruhstorfer, 1908) & $\mathrm{X}$ & - \\
\hline Tatochila autodice autodice (Hübner, 1818) & $\mathrm{X}$ & $\mathrm{X}$ \\
\hline Theochila maenacte maenacte (Boisduval, 1836) & $\mathrm{X}$ & $\mathrm{X}$ \\
\hline \multicolumn{3}{|l|}{ Lycaenidae } \\
\hline \multicolumn{3}{|l|}{ Theclinae } \\
\hline \multicolumn{3}{|l|}{ Eumaeini } \\
\hline Arawacus binangula (Schaus, 1902) & $\mathrm{X}$ & $\mathrm{X}$ \\
\hline Arawacus dolylas (Cramer, 1777) & - & $\mathrm{X}$ \\
\hline Arawacus ellida (Hewitson, 1867) & $\mathrm{X}$ & $\mathrm{X}$ \\
\hline Arawacus meliboeus (Fabricius, 1793) & $\mathrm{X}$ & $\mathrm{X}$ \\
\hline Arawacus separata (Lathy, 1926) & $\mathrm{X}$ & $\mathrm{X}$ \\
\hline Arawacus tadita (Hewitson, 1877) & $\mathrm{X}$ & $\mathrm{X}$ \\
\hline Arcas ducalis (Westwood, 1852) & $\mathrm{X}$ & $\mathrm{X}$ \\
\hline Atlides atys (Cramer, 1779) & $\mathrm{X}$ & - \\
\hline Atlides cosa (Hewitson, 1867) & - & $\mathrm{X}$ \\
\hline Atlides polama (Schaus, 1902) & $\mathrm{X}$ & $\mathrm{X}$ \\
\hline Aubergina vanessoides (Prittwitz, 1865) & $\mathrm{X}$ & $\mathrm{X}$ \\
\hline Brangas silumena (Hewitson, 1867) & $\mathrm{X}$ & - \\
\hline Brevianta celelata (Hewitson, 1874) & $\mathrm{X}$ & $\mathrm{X}$ \\
\hline Chalybs chloris (Hewitson, 1877) & - & $\mathrm{X}$ \\
\hline Chalybs hassan (Stoll, 1790) & $\mathrm{X}$ & $\mathrm{X}$ \\
\hline Calycopis caulonia (Hewitson, 1877) & $\mathrm{X}$ & $\mathrm{X}$ \\
\hline Celmia uzza (Hewitson, 1873) & - & $\mathrm{X}$ \\
\hline Chlorostrymon simaethis (Drury, 1773) & - & $\mathrm{X}$ \\
\hline Chlorostrymon telea (Hewitson, 1868) & $\mathrm{X}$ & $\mathrm{X}$ \\
\hline Contrafacia imma (Prittwitz, 1865) & $\mathrm{X}$ & $\mathrm{X}$ \\
\hline Contrafacia muattina (Schaus, 1902) & $\mathrm{X}$ & $\mathrm{X}$ \\
\hline Cyanophrys bertha (Jones, 1912) & $\mathrm{X}$ & $\mathrm{X}$ \\
\hline Cyanophrys acaste (Prittwitz, 1865) & $\mathrm{X}$ & $\mathrm{X}$ \\
\hline Cyanophrys longula (Hewitson, 1868) & - & $\mathrm{X}$ \\
\hline Cyanophrys remus (Hewitson, 1868) & $\mathrm{X}$ & $\mathrm{X}$ \\
\hline Dicya dicaea (Hewitson, 1874) & $\mathrm{X}$ & $\mathrm{X}$ \\
\hline Dicya eumorpha (Hayward, 1949) & - & $\mathrm{X}$ \\
\hline
\end{tabular}

\begin{tabular}{|c|c|c|}
\hline \multirow[t]{2}{*}{ *Espécies/Subespécie } & 1947 & 2005 \\
\hline & 2002 & 2010 \\
\hline Erora campa (Jones, 1912) & $\mathrm{X}$ & $\mathrm{X}$ \\
\hline Erora nr. gabina (Godman \& Salvin, 1887) & - & $\mathrm{X}$ \\
\hline Erora biblia (Hewitson, 1868) & - & $\mathrm{X}$ \\
\hline Erora tella (Schaus, 1902) & $\mathrm{X}$ & $\mathrm{X}$ \\
\hline Evenus latreillii (Hewitson, 1865) & $\mathrm{X}$ & $\mathrm{X}$ \\
\hline Evenus regalis (Cramer, 1775) & $\mathrm{X}$ & - \\
\hline Gargina caninius (H. H. Druce, 1907) & $\mathrm{X}$ & $\mathrm{X}$ \\
\hline Iaspis temesa (Hewitson, 1868) & $\mathrm{X}$ & - \\
\hline Ignata norax (Godman \& Salvin, 1887) & - & $\mathrm{X}$ \\
\hline Ipidecla schausi (Godman \& Salvin, 1887) & - & $\mathrm{X}$ \\
\hline Janthecla flosculus (H.H. Druce, 1907) & $\mathrm{X}$ & $\mathrm{X}$ \\
\hline Kolana ergina (Hewitson, 1867) & - & $\mathrm{X}$ \\
\hline Kolana ligurina (Hewitson, 1874) & $\mathrm{X}$ & $\mathrm{X}$ \\
\hline Kolana sp. n. & - & $\mathrm{X}$ \\
\hline Lamprospilus badaca (Hewitson, 1868) & - & $\mathrm{X}$ \\
\hline Lamprospilus nubilum (H.H. Druce, 1907) & $\mathrm{X}$ & $\mathrm{X}$ \\
\hline Lamprospilus sp. & $\mathrm{X}$ & - \\
\hline Laothus phydela (Hewitson, 1867) & $\mathrm{X}$ & $\mathrm{X}$ \\
\hline Lathecla sp. & $\mathrm{X}$ & - \\
\hline Magnastigma hirsuta (Prittwitz, 1865) & - & $\mathrm{X}$ \\
\hline Michaelus ira (Hewitson, 1867) & $\mathrm{X}$ & - \\
\hline Michaelus thordesa (Hewitson, 1867) & - & $\mathrm{X}$ \\
\hline Ministrymon azia (Hewitson, 1873) & $\mathrm{X}$ & $\mathrm{X}$ \\
\hline *Ministrymon fostera (Schaus, 1902) & $\mathrm{X}$ & $\mathrm{X}$ \\
\hline Mithras catrea (Hewitson, 1874) & $\mathrm{X}$ & $\mathrm{X}$ \\
\hline Nesiostrymon calchinia (Hewitson, 1868) & - & $\mathrm{X}$ \\
\hline Nicolaea xorema (Schaus, 1902) & $\mathrm{X}$ & $\mathrm{X}$ \\
\hline Nicolaea cupa (H.H. Druce, 1907) & - & $\mathrm{X}$ \\
\hline Nicolaea torris (H.H. Druce, 1907) & - & $\mathrm{X}$ \\
\hline Ocaria ocrisia (Hewitson, 1868) & $\mathrm{X}$ & $\mathrm{X}$ \\
\hline Ocaria thales (Fabricius, 1793) & $\mathrm{X}$ & $\mathrm{X}$ \\
\hline Olynthus fancia (Jones, 1912) & $\mathrm{X}$ & $X$ \\
\hline Ostrinotes sophocles (Fabricius, 1793) & $\mathrm{X}$ & $\mathrm{X}$ \\
\hline Panthiades hebraeus (Hewitson, 1867) & $\mathrm{X}$ & $\mathrm{X}$ \\
\hline Parrhasius orgia (Hewitson, 1867) & $\mathrm{X}$ & $\mathrm{X}$ \\
\hline Parrhasius polibetes (Stoll, 1781) & - & $\mathrm{X}$ \\
\hline Parrhasius selika (Hewitson, 1874) & $\mathrm{X}$ & $\mathrm{X}$ \\
\hline Pseudolycaena marsyas (Linnaeus, 1758) & $\mathrm{X}$ & $\mathrm{X}$ \\
\hline Rekoa malina (Hewitson, 1867) & $\mathrm{X}$ & $\mathrm{X}$ \\
\hline Rekoa palegon (Cramer, 1780) & $\mathrm{X}$ & $\mathrm{X}$ \\
\hline Siderus eliatha (Hewitson, 1867) & $\mathrm{X}$ & - \\
\hline Siderus giapor (Schaus, 1902) & $\mathrm{X}$ & $\mathrm{X}$ \\
\hline Strephonota elika (Hewitson, 1867) & $\mathrm{X}$ & $\mathrm{X}$ \\
\hline Strymon bazochii (Godart, [1824]) & $\mathrm{X}$ & $\mathrm{X}$ \\
\hline Strymon cestri (Reakirt, [1867]) & - & $\mathrm{X}$ \\
\hline Strymon eurytulus (Hübner, [1819]) & $\mathrm{X}$ & $\mathrm{X}$ \\
\hline Strymon lucena (Hewitson, 1868) & $\mathrm{X}$ & $\mathrm{X}$ \\
\hline Strymon sp. & $\mathrm{X}$ & - \\
\hline Strymon sp. & $\mathrm{X}$ & - \\
\hline Strymon oreala (Hewitson, 1868) & $\mathrm{X}$ & $\mathrm{X}$ \\
\hline Strymon rana (Schaus, 1902) & $\mathrm{X}$ & - \\
\hline Strymon yojoa (Reakirt, [1867]) & - & $\mathrm{X}$ \\
\hline
\end{tabular}


Tabela 1. Continuação...

\begin{tabular}{|c|c|c|}
\hline \multirow[t]{2}{*}{ *Espécies/Subespécie } & 1947 & 2005 \\
\hline & 2002 & 2010 \\
\hline Thaeides theia (Hewitson, 1870) & $\mathrm{X}$ & - \\
\hline Thepytus thyrea (Hewitson, 1867) & $\mathrm{X}$ & - \\
\hline Thereus cithonius (Godart, [1824]) & $\mathrm{X}$ & $\mathrm{X}$ \\
\hline Thereus eryssus (Herbst, 1800) & $\mathrm{X}$ & - \\
\hline Thereus ortalus (Godman \& Salvin, 1887) & - & $\mathrm{X}$ \\
\hline Theritas chaluma (Schaus, 1902) & - & $\mathrm{X}$ \\
\hline Theritas deniva (Hewitson, 1874) & $\mathrm{X}$ & $\mathrm{X}$ \\
\hline Theritas hemon (Cramer, 1775) & $\mathrm{X}$ & - \\
\hline Theritas triquetra (Hewitson, 1865) & $\mathrm{X}$ & $\mathrm{X}$ \\
\hline Thestius lycabas (Cramer, 1777) & $\mathrm{X}$ & - \\
\hline Tmolus cydrara (Hewitson, 1868) & $\mathrm{X}$ & - \\
\hline Tmolus echion (Linnaeus, 1767) & $\mathrm{X}$ & $\mathrm{X}$ \\
\hline \multicolumn{3}{|l|}{ Polyommatinae } \\
\hline Elkalyce cogina (Schaus, 1902) & $\mathrm{X}$ & $\mathrm{X}$ \\
\hline Hemiargus hanno (Stoll, 1790) & $\mathrm{X}$ & $\mathrm{X}$ \\
\hline Leptotes cassius cassius (Cramer, 1775) & $\mathrm{X}$ & $\mathrm{X}$ \\
\hline *Pseudolucia parana Bálint, 1993 & $\mathrm{X}$ & $\mathrm{X}$ \\
\hline Zizula cyna (W.H. Edwards, 1881) & $\mathrm{X}$ & $\mathrm{X}$ \\
\hline \multicolumn{3}{|l|}{ Riodinidae } \\
\hline \multicolumn{3}{|l|}{ Euselasiinae } \\
\hline \multicolumn{3}{|l|}{ Euselasiini } \\
\hline Euselasia crinon Stichel, 1919 & - & $\mathrm{X}$ \\
\hline Euselasia eucerus (Hewitson, 1872) & $\mathrm{X}$ & $\mathrm{X}$ \\
\hline Euselasia hygenius occulta Stichel, 1919 & $\mathrm{X}$ & $\mathrm{X}$ \\
\hline Euselasia zara (Westwood, 1851) & - & $\mathrm{X}$ \\
\hline \multicolumn{3}{|l|}{ Riodininae } \\
\hline \multicolumn{3}{|l|}{ Mesosemiini } \\
\hline Ionotos alector (Geyer, 1837) & - & $\mathrm{X}$ \\
\hline Napaea nepos (Fabricius, 1793) & $\mathrm{X}$ & $\mathrm{X}$ \\
\hline Leucochimona icare matatha (Hewitson, 1873) & - & $\mathrm{X}$ \\
\hline Mesosemia friburgensis Schaus 1902 & $\mathrm{X}$ & $\mathrm{X}$ \\
\hline Mesosemia moesia Hewitson, [1857] & $\mathrm{X}$ & - \\
\hline Mesosemia odice (Godart, [1824]) & $\mathrm{X}$ & $\mathrm{X}$ \\
\hline Mesosemia rhodia (Godart, [1824]) & $\mathrm{X}$ & - \\
\hline \multicolumn{3}{|l|}{ Eurybiini } \\
\hline Eurybia halimede (Hübner, [1807]) & - & $\mathrm{X}$ \\
\hline Eurybia pergaea (Geyer, 1832) & - & $\mathrm{X}$ \\
\hline \multicolumn{3}{|l|}{ Riodinini } \\
\hline Baeotis hisbon (Cramer, 1775) & $\mathrm{X}$ & $\mathrm{X}$ \\
\hline Baeotis melanis Hübner, [1831] & $\mathrm{X}$ & - \\
\hline Barbicornis basilis mona Westwood, 1851 & $\mathrm{X}$ & $\mathrm{X}$ \\
\hline Brachyglenis drymo (Godman \& Salvin, 1886) & $\mathrm{X}$ & $\mathrm{X}$ \\
\hline Calephelis braziliensis McAlpine, 1971 & $\mathrm{X}$ & - \\
\hline Calephelis nilus (C. Felder \& R. Felder, 1861) & $\mathrm{X}$ & $\mathrm{X}$ \\
\hline Caria plutargus (Fabricius, 1793) & $\mathrm{X}$ & $\mathrm{X}$ \\
\hline Chalodeta theodora (C. Felder \& R. Felder, 1862) & $\mathrm{X}$ & $\mathrm{X}$ \\
\hline Chamaelimnas briola doryphora Stichel, 1910 & $\mathrm{X}$ & $\mathrm{X}$ \\
\hline Charis cadytis Hewitson, 1866 & $\mathrm{X}$ & $\mathrm{X}$ \\
\hline Chorinea licursis (Fabricius, 1775) & $\mathrm{X}$ & $\mathrm{X}$ \\
\hline Dachetola azora (Godart, [1824]) & - & $\mathrm{X}$ \\
\hline Lasaia agesilas agesilas (Latreille, [1809]) & $\mathrm{X}$ & $\mathrm{X}$ \\
\hline Lasaia incoides (Schaus, 1902) & - & $\mathrm{X}$ \\
\hline
\end{tabular}

\begin{tabular}{|c|c|c|}
\hline \multirow[t]{2}{*}{ *Espécies/Subespécie } & 1947 & 2005 \\
\hline & 2002 & 2010 \\
\hline Pheles atricolor atricolor (Butler, 1871) & - & $\mathrm{X}$ \\
\hline Melanis aegates (Hewitson, 1874) & $\mathrm{X}$ & - \\
\hline Melanis smithiae smithiae (Westwood, 1851) & $\mathrm{X}$ & $\mathrm{X}$ \\
\hline Melanis xenia xenia (Hewitson, [1853]) & $\mathrm{X}$ & $\mathrm{X}$ \\
\hline Monethe alphonsus (Fabricius, 1793) & $\mathrm{X}$ & $\mathrm{X}$ \\
\hline Notheme erota angellus Stichel, 1910 & $\mathrm{X}$ & - \\
\hline Panara soana soana Hewitson, 1875 & $\mathrm{X}$ & $\mathrm{X}$ \\
\hline Parcella amarynthina (C. Felder \& R. Felder, 1865) & $\mathrm{X}$ & $\mathrm{X}$ \\
\hline Rethus periander eleusinus Stichel, 1910 & $\mathrm{X}$ & $\mathrm{X}$ \\
\hline Riodina lycisca (Hewitson, [1853]) & $\mathrm{X}$ & $\mathrm{X}$ \\
\hline Riodina lysippoides Berg, 1882 & $\mathrm{X}$ & $\mathrm{X}$ \\
\hline Syrmatia nyx (Hübner, [1817]) & - & $\mathrm{X}$ \\
\hline \multicolumn{3}{|l|}{ Symmachiini } \\
\hline Mesene epaphus epaphus (Stoll, 1780) & $\mathrm{X}$ & - \\
\hline Mesene pyrippe sanguilenta Stichel, 1910 & $\mathrm{X}$ & $\mathrm{X}$ \\
\hline Pirascca sagaris phrygiana (Stichel, 1916) & $\mathrm{X}$ & $\mathrm{X}$ \\
\hline Stichelia bocchoris suavis (Stichel, 1911) & $\mathrm{X}$ & $\mathrm{X}$ \\
\hline *Stichelia dunkinfieldia (Schaus, 1902) & $\mathrm{X}$ & - \\
\hline Symmachia aconia Hewitson, 1867 & - & $\mathrm{X}$ \\
\hline Symmachia menetas eurima Schaus, 1902 & - & $\mathrm{X}$ \\
\hline \multicolumn{3}{|l|}{ Helicopini } \\
\hline Anteros formosus formosus (Cramer, 1777) & - & $\mathrm{X}$ \\
\hline \multicolumn{3}{|l|}{ Tribo Sedis Incertae } \\
\hline Apodemia castanea (Prittwitz, 1865) & $\mathrm{X}$ & $\mathrm{X}$ \\
\hline Calydna hiria (Godart, [1824]) & $\mathrm{X}$ & $\mathrm{X}$ \\
\hline Calydna sturnula (Geyer, 1837) & $\mathrm{X}$ & - \\
\hline Emesis diogenia Prittwitz, 1865 & - & $\mathrm{X}$ \\
\hline Emesis fatimella fatimella Westwood, 1851 & $\mathrm{X}$ & $\mathrm{X}$ \\
\hline Emesis mandana mandana (Cramer, 1780) & $\mathrm{X}$ & - \\
\hline Emesis neemias Hewitson, 1872 & $\mathrm{X}$ & - \\
\hline Emesis ocypore zelotes Hewitson, 1872 & $\mathrm{X}$ & $\mathrm{X}$ \\
\hline Emesis russula Stichel, 1910 & - & $\mathrm{X}$ \\
\hline Emesis satema (Schaus, 1902) & $\mathrm{X}$ & $\mathrm{X}$ \\
\hline Emesis sp & $\mathrm{X}$ & - \\
\hline Pseudotinea hemis (Schaus, 1927) & $\mathrm{X}$ & $\mathrm{X}$ \\
\hline \multicolumn{3}{|l|}{ Nymphidiini } \\
\hline Adelotypa bolena (Butler, 1867) & $\mathrm{X}$ & $\mathrm{X}$ \\
\hline Adelotypa sejuncta (Stichel, 1910) & $\mathrm{X}$ & $\mathrm{X}$ \\
\hline *Aricoris cinericea $($ Stichel, 1910) & $\mathrm{X}$ & - \\
\hline Aricoris sp. & $\mathrm{X}$ & - \\
\hline Aricoris sp. & $\mathrm{X}$ & - \\
\hline Calospila lucianus lucianus (Fabricius, 1793) & $\mathrm{X}$ & - \\
\hline *Lemonias albofasciata (Godman, 1903) & $\mathrm{X}$ & - \\
\hline *Lemonias ochracea (Mengel, 1902) & $\mathrm{X}$ & - \\
\hline Mycastor leucarpis (Stichel, 1925) & $\mathrm{X}$ & - \\
\hline *Synargis sp. & $\mathrm{X}$ & - \\
\hline Synargis axenus axenus (Hewitson, 1876) & $\mathrm{X}$ & - \\
\hline *Synargis bifasciata (Mengel, 1902) & $\mathrm{X}$ & $\mathrm{X}$ \\
\hline Synargis calyce (C. Felder \& R. Felder, 1862) & $\mathrm{X}$ & - \\
\hline Synargis phliasus phliasus (Clerck, 1764) & $\mathrm{X}$ & $\mathrm{X}$ \\
\hline Synargis paulistina (Stichel, 1910) & $\mathrm{X}$ & - \\
\hline Synargis regulus (Fabricius, 1793) & $\mathrm{X}$ & - \\
\hline
\end{tabular}


Tabela 1. Continuação...

\begin{tabular}{|c|c|c|}
\hline \multirow[t]{2}{*}{ *Espécies/Subespécie } & \multirow{2}{*}{$\frac{1947}{2002}$} & \multirow{2}{*}{$\begin{array}{l}2005 \\
2010\end{array}$} \\
\hline & & \\
\hline Theope thestias Hewitson, 1860 & $\mathrm{X}$ & $\mathrm{X}$ \\
\hline \multicolumn{3}{|l|}{ Nymphalidae } \\
\hline \multicolumn{3}{|l|}{ Libytheinae } \\
\hline Libytheana carinenta (Cramer, 1777) & $\mathrm{X}$ & $\mathrm{X}$ \\
\hline \multicolumn{3}{|l|}{ Danainae } \\
\hline \multicolumn{3}{|l|}{ Euploeini } \\
\hline Lycorea halia discreta Haensch, 1909 & $\mathrm{X}$ & $\mathrm{X}$ \\
\hline Lycorea ilione ilione (Cramer, 1775) & $\mathrm{X}$ & $\mathrm{X}$ \\
\hline \multicolumn{3}{|l|}{ Danaini } \\
\hline Danaus eresimus plexaure (Godart, 1819) & $\mathrm{X}$ & $\mathrm{X}$ \\
\hline Danaus erippus (Cramer, 1775) & $\mathrm{X}$ & $\mathrm{X}$ \\
\hline Danaus gilippus gilippus (Cramer, 1775) & $\mathrm{X}$ & $\mathrm{X}$ \\
\hline \multicolumn{3}{|l|}{ Ithomiinae } \\
\hline \multicolumn{3}{|l|}{ Tithoreini } \\
\hline Aeria olena olena Weymer, 1875 & $\mathrm{X}$ & $\mathrm{X}$ \\
\hline \multicolumn{3}{|l|}{ Mechanitini } \\
\hline Mechanitis lysimnia lysimnia (Fabricius, 1793) & $\mathrm{X}$ & $\mathrm{X}$ \\
\hline Mechanitis polymnia casabranca Haensch, 1905 & $\mathrm{X}$ & $\mathrm{X}$ \\
\hline Methona themisto themisto (Hübner, 1818) & $\mathrm{X}$ & $\mathrm{X}$ \\
\hline Thyridia psidii cetoides (Rosenberg \& Talbot, 1914) & $\mathrm{X}$ & $\mathrm{X}$ \\
\hline \multicolumn{3}{|l|}{ Napeogenini } \\
\hline Epityches eupompe (Geyer, 1832) & $\mathrm{X}$ & $\mathrm{X}$ \\
\hline Hypothyris euclea laphria (Doubleday, 1847) & $\mathrm{X}$ & $\mathrm{X}$ \\
\hline Hypothyris ninonia daeta (Boisduval, 1836) & $\mathrm{X}$ & $\mathrm{X}$ \\
\hline \multicolumn{3}{|l|}{ Ithomiini } \\
\hline Placidina euryanassa (C. Felder \& R. Felder, 1860) & $\mathrm{X}$ & $\mathrm{X}$ \\
\hline Ithomia agnosia zikani d'Almeida, 1940 & $\mathrm{X}$ & $\mathrm{X}$ \\
\hline Ithomia drymo Hübner, 1816 & $\mathrm{X}$ & $\mathrm{X}$ \\
\hline Ithomia lichyi lichyi d'Almeida, 1939 & $\mathrm{X}$ & $\mathrm{X}$ \\
\hline \multicolumn{3}{|l|}{ Dircennini } \\
\hline Dircenna dero dero (Hübner, 1823) & $\mathrm{X}$ & $\mathrm{X}$ \\
\hline Episcada carcinia Schaus, 1902 & $\mathrm{X}$ & $\mathrm{X}$ \\
\hline Episcada hymenaea hymenaea (Prittwitz, 1865) & $\mathrm{X}$ & $\mathrm{X}$ \\
\hline Episcada philoclea (Hewitson, [1855]) & $\mathrm{X}$ & - \\
\hline Hyalenna pascua (Schaus, 1902) & - & $\mathrm{X}$ \\
\hline Pteronymia sylvo (Geyer, 1832) & $\mathrm{X}$ & $\mathrm{X}$ \\
\hline \multicolumn{3}{|l|}{ Godyridini } \\
\hline Brevioleria seba emyra (Haensch, 1905) & - & $\mathrm{X}$ \\
\hline Mcclungia cymo salonina (Hewitson, 1855) & $\mathrm{X}$ & $\mathrm{X}$ \\
\hline Pseudoscada erruca (Hewitson, 1855) & $\mathrm{X}$ & $\mathrm{X}$ \\
\hline \multicolumn{3}{|l|}{ Morphinae } \\
\hline \multicolumn{3}{|l|}{ Morphini } \\
\hline Cytheritis aega aega (Hübner, [1822]) & $\mathrm{X}$ & $\mathrm{X}$ \\
\hline Cytheritis portis thamyris (C. Felder \& R. Felder, 1867) & $\mathrm{X}$ & $\mathrm{X}$ \\
\hline Iphixibia anaxibia (Esper, [1801]) & $\mathrm{X}$ & $\mathrm{X}$ \\
\hline Morpho helenor achillaena (Hübner, [1823]) & $\mathrm{X}$ & $\mathrm{X}$ \\
\hline Pessonia epistrophus catenaria (Perry, 1811) & $\mathrm{X}$ & $\mathrm{X}$ \\
\hline \multicolumn{3}{|l|}{ Brassolini } \\
\hline Blepolenis bassus (C. Felder \& R. Felder, 1867) & $\mathrm{X}$ & $\mathrm{X}$ \\
\hline Blepolenis batea (Hübner, [1821]) & $\mathrm{X}$ & $\mathrm{X}$ \\
\hline Brassolis astyra philocala Stichel, 1904 & $\mathrm{X}$ & $\mathrm{X}$ \\
\hline Caligo illioneus pampeiro Fruhstorfer, 1904 & - & $\mathrm{X}$ \\
\hline
\end{tabular}

\begin{tabular}{lcc}
\hline \multicolumn{1}{c}{ *Espécies/Subespécie } & $\mathbf{1 9 4 7}$ & $\mathbf{2 0 0 5}$ \\
\cline { 2 - 4 } & $\mathbf{2 0 0 2}$ & $\mathbf{2 0 1 0}$ \\
\hline Caligo martia (Godart, [1824]) & $\mathrm{X}$ & $\mathrm{X}$ \\
Catoplepia amphirhoe (Hübner, [1825]) & $\mathrm{X}$ & $\mathrm{X}$ \\
Dynastor darius (Fabricius, 1775) & $\mathrm{X}$ & $\mathrm{X}$ \\
Dynastor napoleon Doubleday, [1849] & $\mathrm{X}$ & - \\
Eryphanis reevesii Doubleday, [1849]) & $\mathrm{X}$ & $\mathrm{X}$ \\
Narope cyllastros Doubleday, [1849] & $\mathrm{X}$ & - \\
Narope cyllene C. Felder \& R. Felder, 1859 & $\mathrm{X}$ & $\mathrm{X}$ \\
Narope panniculus Stichel, 1904 & $\mathrm{X}$ & - \\
Opoptera aorsa aorsa (Godart, [1824]) & $\mathrm{X}$ & - \\
Opoptera fruhstorferi (Röber, 1896) & $\mathrm{X}$ & $\mathrm{X}$ \\
Opoptera sulcius (Staudinger, 1887) & $\mathrm{X}$ & $\mathrm{X}$ \\
Opsiphanes invirae amplificatus Stichel, 1904 & $\mathrm{X}$ & $\mathrm{X}$ \\
Penetes pamphanis Doubleday, [1849] & $\mathrm{X}$ & $\mathrm{X}$
\end{tabular}

Satyrinae

Elymniini

Manataria hercyna hercyna (Hübner, [1821]) $\quad$ X X

Satyrini

$\begin{array}{lll}\text { Capronnieria galesus (Godart, [1824]) } & \text { X } & \text { X }\end{array}$

*Erichthodes narapa (Schaus, 1902) X X

Eteona tisiphone (Boisduval, 1836) $\quad \mathrm{X} \quad \mathrm{X}$

Euptychoides castrensis (Schaus, 1902) $\quad$ X $\quad$ X

Forsterinaria necys (Godart, [1824]) $\quad$ X $\quad$ X

Forsterinaria quantius (Godart, [1824]) $\quad$ X $\quad$ X

Godartiana muscosa (Butler, 1870) $\quad$ X X

Guaianaza pronophila (Butler, 1867) X X

Hermeuptychia hermes (Fabricius, 1775) X X X

Carminda griseldis (Weymer, 1911) X X

Carminda paeon (Godart, [1824]) $\quad$ X $\quad$ X

Carminda soter (Butler, 1877) $\quad \mathrm{X} \quad \mathrm{X}$

*Moneuptychia sp. n. $\quad$ X X

*Pampasatyrus glaucope glaucope (C. Felder \& R. Felder, X 1867)

*Pampasatyrus ocelloides (Schaus, 1902) $\quad$ X $\quad$ X

*Pampasatyrus periphas (Godart, [1824]) $\quad$ X X

*Pampasatyrus sp.

Pareuptychia ocirrhoe interjecta (d'Almeida, 1952) X -

Pareuptychia summandosa (Gosse, 1880) $\quad$ X X

Paryphthimoides eous (Butler, 1867) - $\quad \mathrm{X}$

Paryphthimoides grimon (Godart, [1824]) X X X

Paryphthimoides numeria (C. Felder \& R. Felder, 1867) X -

Paryphthimoides phronius (Godart, [1824]) X X

Paryphthimoides poltys (Prittwitz, 1865) X X X

Praepedaliodes phanias (Hewitson, 1862) X X X

Splendeuptychia cosmophila (Hübner, 1823) - $\mathrm{X}$

Splendeuptychia hygina (Butler, 1877) X X

Splendeuptychia libitina (Butler, 1870) - $\mathrm{X}$

Taydebis peculiaris (Butler, 1874) $\quad$ X $\quad$ X

Taygetis acuta Weyer, $1910 \quad$ X -

Taygetis laches marginata Staudinger, [1887] $\quad$ X X

Taygetis rufomarginata Staudinger, $1888 \quad$ X X

Taygetis tripunctata Weymer, 1907 - $\mathrm{X}$

Taygetis ypthima Hübner, [1821] $\quad$ X $\quad$ X 
Tabela 1. Continuação...

\begin{tabular}{|c|c|c|}
\hline \multirow[t]{2}{*}{ *Espécies/Subespécie } & 1947 & 2005 \\
\hline & 2002 & 2010 \\
\hline Yphthimoides affinis (Butler, 1867) & - & $\mathrm{X}$ \\
\hline Yphthimoides angularis (Butler, 1867) & $\mathrm{X}$ & $\mathrm{X}$ \\
\hline Yphthimoides celmis (Godart, [1824]) & $\mathrm{X}$ & $\mathrm{X}$ \\
\hline Yphthimoides ochracea (Butler, 1867) & $\mathrm{X}$ & $\mathrm{X}$ \\
\hline Yphthimoides renata $($ Stoll, 1780$)$ & - & $\mathrm{X}$ \\
\hline Yphthimoides sp. n. & - & $\mathrm{X}$ \\
\hline Yphthimoides straminea (Butler, 1867) & $\mathrm{X}$ & $\mathrm{X}$ \\
\hline Yphthimoides viviana (Romieux, 1927) & $\mathrm{X}$ & $\mathrm{X}$ \\
\hline Yphthimoides yphthima (C. Felder \& R. Felder, 1867) & $\mathrm{X}$ & $\mathrm{X}$ \\
\hline Zischkaia pacarus (Godart, [1824]) & $\mathrm{X}$ & $\mathrm{X}$ \\
\hline \multicolumn{3}{|l|}{ Charaxinae } \\
\hline \multicolumn{3}{|l|}{ Anaeini } \\
\hline Consul fabius drurii (Butler, 1874) & $\mathrm{X}$ & - \\
\hline Fountainea ryphea phidile (Geyer, 1837) & $\mathrm{X}$ & $\mathrm{X}$ \\
\hline Hypna clytemnestra huebneri Butler, 1866 & $\mathrm{X}$ & $\mathrm{X}$ \\
\hline Memphis acidalia victoria $(\mathrm{H}$. Druce, 1877$)$ & $\mathrm{X}$ & $\mathrm{X}$ \\
\hline Memphis hirta (Weymer, 1907) & $\mathrm{X}$ & $\mathrm{X}$ \\
\hline Memphis moruus stheno (Prittwitz, 1865) & $\mathrm{X}$ & $\mathrm{X}$ \\
\hline Memphis otrere (Hübner, [1825]) & $\mathrm{X}$ & - \\
\hline Zaretis itys itylus (Westwood, 1850) & $\mathrm{X}$ & $\mathrm{X}$ \\
\hline \multicolumn{3}{|l|}{ Preponini } \\
\hline $\begin{array}{l}\text { Archaeoprepona amphimachus pseudomeander } \\
\text { (Fruhstorfer, 1906) }\end{array}$ & $\mathrm{X}$ & $\mathrm{X}$ \\
\hline Archaeoprepona chalciope (Hübner, [1823]) & $\mathrm{X}$ & $\mathrm{X}$ \\
\hline Archaeoprepona demophon thalpius (Hübner, [1814]) & $\mathrm{X}$ & $\mathrm{X}$ \\
\hline Archaeoprepona demophoon demophoon (Hübner, 1814) & $\mathrm{X}$ & $\mathrm{X}$ \\
\hline Prepona pylene Hewitson, [1854] & $\mathrm{X}$ & $\mathrm{X}$ \\
\hline \multicolumn{3}{|l|}{ Biblidinae } \\
\hline \multicolumn{3}{|l|}{ Cyrestini } \\
\hline Marpesia chiron marius (Cramer, 1779) & - & $\mathrm{X}$ \\
\hline Marpesia petreus petreus (Cramer, 1776) & $\mathrm{X}$ & $\mathrm{X}$ \\
\hline \multicolumn{3}{|l|}{ Biblidini } \\
\hline Biblis hyperia nectanabis (Fruhstorfer, 1909) & $\mathrm{X}$ & $\mathrm{X}$ \\
\hline Callicore hydaspes (Drury, 1782) & $\mathrm{X}$ & $\mathrm{X}$ \\
\hline Callicore pygas eucale (Fruhstorfer, 1916) & $\mathrm{X}$ & $\mathrm{X}$ \\
\hline Callicore sorana sorana (Godart, [1824]) & $\mathrm{X}$ & - \\
\hline Catonephele acontius acontius (Linnaeus, 1771) & $\mathrm{X}$ & - \\
\hline Catonephele numilia penthia (Hewitson, 1852) & $\mathrm{X}$ & $\mathrm{X}$ \\
\hline Catonephele sabrina (Hewitson, 1852) & $\mathrm{X}$ & - \\
\hline Cybdelis phaesyla (Hübner, [1831]) & $\mathrm{X}$ & $\mathrm{X}$ \\
\hline Diaethria candrena candrena (Godart, [1824]) & $\mathrm{X}$ & $\mathrm{X}$ \\
\hline Diaethria clymena meridionalis (H.W. Bates, 1864) & $\mathrm{X}$ & $\mathrm{X}$ \\
\hline Diaethria eluina eluina (Hewitson, [1855]) & $\mathrm{X}$ & $\mathrm{X}$ \\
\hline Dynamine agacles agacles (Dalman, 1823) & $\mathrm{X}$ & $\mathrm{X}$ \\
\hline Dynamine artemisia artemisia (Fabricius, 1793) & $\mathrm{X}$ & $\mathrm{X}$ \\
\hline Dynamine athemon athemon (Linnaeus, 1758) & $\mathrm{X}$ & $\mathrm{X}$ \\
\hline Dynamine myrrhina (Doubleday, 1849) & $\mathrm{X}$ & $\mathrm{X}$ \\
\hline Dynamine postverta postverta (Cramer, 1779) & $\mathrm{X}$ & $\mathrm{X}$ \\
\hline Dynamine tithia tithia (Hübner, 1823) & $\mathrm{X}$ & $\mathrm{X}$ \\
\hline Ectima thecla thecla (Fabricius, 1796) & $\mathrm{X}$ & $X$ \\
\hline Epiphile hubneri Hewitson, 1861 & $\mathrm{X}$ & $\mathrm{X}$ \\
\hline Epiphile orea orea (Hübner, [1823]) & $\mathrm{X}$ & $\mathrm{X}$ \\
\hline
\end{tabular}

\begin{tabular}{|c|c|c|}
\hline \multirow[t]{2}{*}{ *Espécies/Subespécie } & 1947 & 2005 \\
\hline & 2002 & 2010 \\
\hline Eunica caelina caelina (Godart, [1824]) & $\mathrm{X}$ & $\mathrm{X}$ \\
\hline Eunica eburnea Fruhstorfer, 1907 & $\mathrm{X}$ & $\mathrm{X}$ \\
\hline Eunica maja maja (Fabricius, 1775) & $\mathrm{X}$ & - \\
\hline Eunica margarita (Godart, [1824]) & - & $\mathrm{X}$ \\
\hline Eunica tatila bellaria Fruhstorfer, 1908 & $\mathrm{X}$ & $\mathrm{X}$ \\
\hline Haematera pyrame pyrame Hübner, [1819] & $\mathrm{X}$ & $\mathrm{X}$ \\
\hline Hamadryas amphinome amphinome (Linnaeus, 1767) & $\mathrm{X}$ & $\mathrm{X}$ \\
\hline Hamadryas arete (Doubleday, 1847) & $\mathrm{X}$ & - \\
\hline Hamadryas epinome (C. Felder \& R. Felder, 1867) & $\mathrm{X}$ & $\mathrm{X}$ \\
\hline Hamadryas februa februa (Hübner, [1823]) & $\mathrm{X}$ & $\mathrm{X}$ \\
\hline Hamadryas feronia feronia (Linnaeus, 1758) & $\mathrm{X}$ & $\mathrm{X}$ \\
\hline Hamadryas fornax fornax (Hübner, [1823]) & $\mathrm{X}$ & $\mathrm{X}$ \\
\hline Hamadryas iphthime iphthime (H.W. Bates, 1864) & $\mathrm{X}$ & $\mathrm{X}$ \\
\hline Mestra dorcas apicalis (Staudinger, 1886) & $\mathrm{X}$ & - \\
\hline Myscelia orsis (Drury, 1782) & $\mathrm{X}$ & $\mathrm{X}$ \\
\hline Paulogramma pyracmon pyracmon (Godart, [1824]) & $\mathrm{X}$ & $\mathrm{X}$ \\
\hline Pyrrhogyra neaerea arge Gosse, 1880 & $\mathrm{X}$ & - \\
\hline Temenis laothoe meridionalis Ebert, 1965 & $\mathrm{X}$ & $\mathrm{X}$ \\
\hline \multicolumn{3}{|l|}{ Apaturinae } \\
\hline Doxocopa agathina vacuna (Godart, [1824]) & $\mathrm{X}$ & $\mathrm{X}$ \\
\hline Doxocopa kallina (Staudinger, 1886) & $\mathrm{X}$ & $\mathrm{X}$ \\
\hline Doxocopa laurentia laurentia (Godart, [1824]) & $\mathrm{X}$ & $\mathrm{X}$ \\
\hline Doxocopa zunilda zunilda (Godart, [1824]) & $\mathrm{X}$ & $\mathrm{X}$ \\
\hline
\end{tabular}

Nymphalinae

Coeini

Colobura dirce dirce (Linnaeus, 1758)

Historis odius odius (Fabricius, 1775)

Smyrna blomfildia blomfildia (Fabricius, 1781)

Nymphalini

Hypanartia bella (Fabricius, 1793)

Hypanartia lethe (Fabricius, 1793)

Vanessa braziliensis (Moore, 1883)

Vanessa carye (Hübner, [1812])

Vanessa myrinna (Doubleday, 1849)

Kallimini

Anarthia amathea roeselia (Eschscholtz, 1821)

Anartia jatrophae jatrophae (Linnaeus, 1763)

Junonia evarete evarete (Cramer, 1779)

Siproeta epaphus trayja Hübner, [1823]

Siproeta stelenes meridionalis (Fruhstorfer, 1909)

Melitaeini

Chlosyne lacinia saundersi (Doubleday, [1847])

Eresia lansdorfi (Godart, 1819)

Ortilia dicoma (Hewitson, 1864)

Ortilia ithra (W.F. Kirby, 1900)

Ortilia orthia (Hewitson, 1864)

Ortilia velica durnfordi (Godman \& Salvin, 1878)

Tegosa claudina (Eschscholtz, 1821)

Tegosa orobia orobia (Hewitson, 1864)

Telenassa teletusa teletusa (Godart, [1824])

Limenitidinae

Adelpha abia (Hewitson, 1850)

\begin{tabular}{ll}
$X$ & $X$ \\
$X$ & $X$ \\
$X$ & $X$ \\
& \\
$X$ & $X$ \\
$X$ & $X$ \\
$X$ & $X$ \\
$X$ & $X$ \\
$X$ & $X$ \\
& \\
$X$ & $X$ \\
$X$ & - \\
$X$ & $X$ \\
$X$ & $X$ \\
$X$ & $X$ \\
& \\
$X$ & $X$ \\
$X$ & $X$ \\
$X$ & $X$ \\
$X$ & $X$ \\
$X$ & $X$ \\
$X$ & $X$ \\
$X$ & $X$ \\
$X$ & $X$ \\
$X$ & $X$ \\
& \\
- & $X$ \\
\hline
\end{tabular}


Tabela 1. Continuação...

\begin{tabular}{lccc}
\hline \multicolumn{1}{c}{ *Espécies/Subespécie } & $\mathbf{1 9 4 7}$ & $\mathbf{2 0 0 5}$ \\
\cline { 2 - 4 } & $\mathbf{2 0 0 2}$ & $\mathbf{2 0 1 0}$ \\
\hline Adelpha calliphane Fruhstorfer, 1915 & $\mathrm{X}$ & - \\
Adelpha cytherea aea (C. Felder \& R. Felder, 1867) & $\mathrm{X}$ & - \\
Adelpha falcipennis Fruhstorfer, 1915 & $\mathrm{X}$ & - \\
Adelpha gavina Fruhstorfer, 1915 & $\mathrm{X}$ & $\mathrm{X}$ \\
Adelpha hyas hyas (Doyère, [1840]) & - & $\mathrm{X}$ \\
Adelpha malea goyama Schaus, 1902 & $\mathrm{X}$ & $\mathrm{X}$ \\
Adelpha mythra (Godart, [1824]) & - & $\mathrm{X}$ \\
Adelpha poltius A. Hall, 1938 & - & $\mathrm{X}$ \\
Adelpha radiata radiata Fruhstorfer, 1915 & $\mathrm{X}$ & - \\
Adelpha serpa serpa (Boisduval, 1836) & $\mathrm{X}$ & - \\
Adelpha syma (Godart, [1824]) & $\mathrm{X}$ & $\mathrm{X}$ \\
Adelpha thesprotia (C. Felder \& R. Felder, 1867) & - & $\mathrm{X}$ \\
Adelpha thessalia indefecta Fruhstorfer, 1913 & $\mathrm{X}$ & $\mathrm{X}$ \\
Adelpha zea (Hewitson, 1850) & - & $\mathrm{X}$ \\
Heliconiinae & & \\
Argynnini & & \\
Euptoieta hegesia meridiania Stichel, 1938 & $\mathrm{X}$ & $\mathrm{X}$ \\
Euptoieta hortensia (Blanchard, 1852) & $\mathrm{X}$ & - \\
Acraeini & & \\
Actinote alalia (C. Felder \& R. Felder, 1860) & $\mathrm{X}$ & $\mathrm{X}$ \\
\hline
\end{tabular}

\begin{tabular}{lcc}
\hline \multicolumn{1}{c}{ *Espécies/Subespécie } & $\mathbf{1 9 4 7}$ & $\mathbf{2 0 0 5}$ \\
\cline { 2 - 4 } & $\mathbf{2 0 0 2}$ & $\mathbf{2 0 1 0}$ \\
\hline Actinote carycina Jordan, 1913 & $\mathrm{X}$ & $\mathrm{X}$ \\
Actinote genitrix d'Almeida, 1922 & - & $\mathrm{X}$ \\
Actinote melanisans Oberthür, 1917 & $\mathrm{X}$ & $\mathrm{X}$ \\
Actinote parapheles Jordan, 1913 & $\mathrm{X}$ & $\mathrm{X}$ \\
Actinote pellenea pellenea Hübner, [1821] & $\mathrm{X}$ & $\mathrm{X}$ \\
Actinote rhodope d'Almeida, 1923 & - & $\mathrm{X}$ \\
Actinote surima surima (Schaus, 1902) & $\mathrm{X}$ & $\mathrm{X}$ \\
Actinote thalia pyrrha (Fabricius, 1775) & $\mathrm{X}$ & $\mathrm{X}$ \\
Heliconiini & & \\
Agraulis vanille maculosa (Stichel, [1908]) & $\mathrm{X}$ & $\mathrm{X}$ \\
Dione juno juno (Cramer, [1779]) & $\mathrm{X}$ & $\mathrm{X}$ \\
Dione moneta moneta Hübner, [1825] & $\mathrm{X}$ & $\mathrm{X}$ \\
Dryadula phaetusa (Linnaeus, 1758) & $\mathrm{X}$ & $\mathrm{X}$ \\
Dryas iulia alcionea (Cramer, 1779) & $\mathrm{X}$ & $\mathrm{X}$ \\
Eueides aliphera aliphera $($ Godart, 1819) & $\mathrm{X}$ & $\mathrm{X}$ \\
Eueides isabella dianasa (Hübner, [1806]) & $\mathrm{X}$ & $\mathrm{X}$ \\
Heliconius besckei Ménétriés, 1857 & $\mathrm{X}$ & $\mathrm{X}$ \\
Heliconius erato phyllis (Fabricius, 1775) & $\mathrm{X}$ & $\mathrm{X}$ \\
Heliconius ethilla narcaea Godart, 1819 & $\mathrm{X}$ & $\mathrm{X}$ \\
Philaethria wernickei (Röber, 1906) & $\mathrm{X}$ & $\mathrm{X}$ \\
\hline
\end{tabular}

Tabela 2. Riqueza dos taxa superiores de borboletas de Guarapuava e arredores. Números em destaque correspondem a valores totais para cada família/ subfamília. Is = índice de Sorensen para a riqueza total em cada taxa entre os dados de 1944 a 2002 e 2005 a 2010.

Table 2. Richness of higher taxa of butterflies from Guarapuava and vicinity. Numbers highlighted represent totals for each family/subfamily. Is $=$ Sörensen index for total richness in each of the data taxa from 1944 to 2002 and from 2005 to 2010.

\begin{tabular}{|c|c|c|c|c|c|c|c|c|}
\hline \multirow[t]{3}{*}{ Taxa } & \multicolumn{2}{|c|}{ Número } & \multirow[t]{3}{*}{$\begin{array}{c}\text { Espécies de Campo } \\
\text { Natural }\end{array}$} & \multicolumn{2}{|c|}{ Amostragens } & \multicolumn{2}{|c|}{$\begin{array}{c}\text { Espécies } \\
\text { exclusivas }\end{array}$} & \multirow[t]{3}{*}{ Is } \\
\hline & Sp. & Spp. & & 1944 & 2005 & 1944 & 2005 & \\
\hline & & & & 2002 & 2010 & 2002 & 2010 & \\
\hline HESPERIOIDEA & 255 & 256 & 26 & 199 & 183 & 73 & 56 & 66 \\
\hline HESPERIIDAE & 255 & 256 & 26 & 199 & 183 & 73 & 56 & 66 \\
\hline Pyrrhopyginae & 11 & 11 & $\mathbf{0}$ & 11 & 9 & 2 & $\mathbf{0}$ & 90 \\
\hline Oxynetrini & 1 & 1 & 0 & 1 & 1 & 0 & 0 & 100 \\
\hline Passovini & 1 & 1 & 0 & 1 & 0 & 1 & 0 & $\mathbf{0}$ \\
\hline Pyrrhopygini & 9 & 9 & 0 & 9 & 8 & 1 & 0 & 94,1 \\
\hline Pyrginae & 95 & 96 & 7 & 74 & 71 & 24 & 21 & 69 \\
\hline Eudamini & 39 & 40 & 1 & 33 & 28 & 11 & 6 & 72,1 \\
\hline Pyrgini & 56 & 56 & 6 & 41 & 43 & 13 & 15 & 66,7 \\
\hline Hesperiinae & 146 & 146 & 19 & 111 & 102 & 45 & 35 & 62 \\
\hline Heteropterinae & 3 & 3 & $\mathbf{0}$ & 3 & 1 & 2 & $\mathbf{0}$ & 50 \\
\hline PAPILIONOIDEA & 434 & 436 & 16 & 375 & 360 & 73 & 61 & 81,6 \\
\hline PAPILIONIDAE & 19 & 21 & 1 & 19 & 15 & 4 & $\mathbf{0}$ & 88,2 \\
\hline Papilioninae & 19 & 21 & 1 & 19 & 15 & 4 & $\mathbf{0}$ & 88,2 \\
\hline Troidini & 7 & 8 & 1 & 7 & 7 & 0 & 0 & 100 \\
\hline Leptocircini & 5 & 6 & 0 & 5 & 2 & 3 & 0 & 57,1 \\
\hline Papilionini & 7 & 7 & 0 & 7 & 6 & 1 & 0 & 92,3 \\
\hline PIERIDAE & 33 & 33 & $\mathbf{0}$ & 32 & 29 & 4 & 1 & 91,8 \\
\hline Dismorphiinae & 7 & 7 & $\mathbf{0}$ & 7 & 6 & 1 & $\mathbf{0}$ & 92,3 \\
\hline Coliadinae & 14 & 14 & 1 & 14 & 13 & 1 & $\mathbf{0}$ & 96,3 \\
\hline Pierinae & 12 & 12 & $\mathbf{0}$ & 11 & 10 & 2 & 1 & 85,7 \\
\hline Anthocharidini & 2 & 2 & 0 & 2 & 2 & 0 & 0 & 100 \\
\hline
\end{tabular}




\begin{tabular}{|c|c|c|c|c|c|c|c|c|}
\hline \multirow[t]{3}{*}{ Taxa } & \multicolumn{2}{|c|}{ Número } & \multirow[t]{3}{*}{$\begin{array}{c}\text { Espécies de Campo } \\
\text { Natural }\end{array}$} & \multicolumn{2}{|c|}{ Amostragens } & \multicolumn{2}{|c|}{$\begin{array}{c}\text { Espécies } \\
\text { exclusivas }\end{array}$} & \multirow[t]{3}{*}{ Is } \\
\hline & Sp. & Spp. & & 1944 & 2005 & 1944 & 2005 & \\
\hline & & & & 2002 & 2010 & 2002 & 2010 & \\
\hline Pierini & 10 & 10 & 0 & 9 & 8 & 2 & 1 & 82,4 \\
\hline LYCAENIDAE & 96 & 96 & 2 & 71 & 79 & 17 & 25 & 72 \\
\hline Theclinae & 91 & 91 & 1 & 66 & 74 & 17 & 25 & 70 \\
\hline Eumaeini & 91 & 91 & 1 & 66 & 74 & 17 & 25 & 70 \\
\hline Polyommatinae & 5 & 5 & 1 & 5 & 5 & $\mathbf{0}$ & $\mathbf{0}$ & 100 \\
\hline RIODINIDAE & 76 & 76 & 7 & 62 & 52 & 22 & 15 & 66,1 \\
\hline Euselasiinae & 4 & 4 & $\mathbf{0}$ & 2 & 4 & $\mathbf{0}$ & 2 & 66,7 \\
\hline Euselasiini & 4 & 4 & 0 & 2 & 4 & 0 & 2 & 66,7 \\
\hline Riodininae & 70 & 70 & 7 & 58 & 48 & 22 & 13 & 68,4 \\
\hline Mesosemiini & 7 & 7 & 0 & 5 & 5 & 2 & 2 & 60 \\
\hline Eurybiini & 2 & 2 & 0 & 1 & 2 & 0 & 2 & 0 \\
\hline Riodinini & 26 & 26 & 0 & 22 & 22 & 4 & 4 & 81,8 \\
\hline Symmachiini & 7 & 7 & 1 & 5 & 5 & 2 & 2 & 60 \\
\hline Helicopini & 1 & 1 & 0 & 0 & 1 & 0 & 1 & $\mathbf{0}$ \\
\hline Tribo Sedis Incertae & 12 & 12 & 0 & 10 & 8 & 4 & 2 & 66,7 \\
\hline Nymphidiini & 17 & 17 & 5 & 17 & 5 & 10 & 0 & 63,6 \\
\hline NYMPHALIDAE & 210 & 210 & 6 & 191 & 185 & 26 & 20 & 87,2 \\
\hline Libytheinae & 1 & 1 & $\mathbf{0}$ & 1 & 1 & $\mathbf{0}$ & $\mathbf{0}$ & 100 \\
\hline Danainae & 5 & 5 & $\mathbf{0}$ & 5 & 5 & $\mathbf{0}$ & $\mathbf{0}$ & 100 \\
\hline Euploeini & 2 & 2 & 0 & 2 & 2 & 0 & 0 & 100 \\
\hline Danaini & 3 & 3 & 0 & 3 & 3 & 0 & 0 & 100 \\
\hline Ithomiinae & 21 & 21 & 0 & 19 & 20 & 1 & 2 & 92,3 \\
\hline Tithoreini & 1 & 1 & 0 & 1 & 1 & 0 & 0 & 100 \\
\hline Mechanitini & 4 & 4 & 0 & 4 & 4 & 0 & 0 & 100 \\
\hline Napeogenini & 3 & 3 & 0 & 3 & 3 & 0 & 0 & 100 \\
\hline Ithomiini & 4 & 4 & 0 & 4 & 4 & 0 & 0 & 100 \\
\hline Dirceniini & 6 & 6 & 0 & 5 & 5 & 1 & 1 & 80 \\
\hline Godyrini & 3 & 3 & 0 & 2 & 3 & 0 & 1 & 80 \\
\hline Morphinae & 22 & 22 & 0 & 21 & 18 & 4 & 1 & 87,2 \\
\hline Morphini & 5 & 5 & 0 & 5 & 5 & 0 & 0 & 100 \\
\hline Brassolini & 17 & 17 & 0 & 16 & 13 & 4 & 1 & 82,8 \\
\hline Satyrinae & 45 & 45 & 6 & 39 & 41 & 5 & 7 & 82,5 \\
\hline Elymniini & 1 & 1 & 0 & 1 & 1 & 0 & 0 & 100 \\
\hline Satyrini & 44 & 44 & 6 & 38 & 40 & 5 & 7 & 82,1 \\
\hline Charaxinae & 13 & 13 & $\mathbf{0}$ & 13 & 11 & 2 & $\mathbf{0}$ & 91,7 \\
\hline Anaeini & 8 & 8 & 0 & 8 & 6 & 2 & 0 & 85,7 \\
\hline Preponini & 5 & 5 & 0 & 5 & 5 & 0 & 0 & 100 \\
\hline Biblidinae & 40 & 40 & $\mathbf{0}$ & 38 & 33 & 7 & 2 & 87,3 \\
\hline Cyrestini & 2 & 2 & 0 & 1 & 2 & 0 & 1 & 66,7 \\
\hline Biblidini & 38 & 38 & 0 & 37 & 31 & 7 & 1 & 88,2 \\
\hline Apaturinae & 4 & 4 & 0 & 4 & 4 & $\mathbf{0}$ & $\mathbf{0}$ & 100 \\
\hline Nymphalinae & 22 & 22 & $\mathbf{0}$ & 22 & 21 & 1 & $\mathbf{0}$ & 97,7 \\
\hline Coeini & 3 & 3 & 0 & 3 & 3 & 0 & 0 & 100 \\
\hline Nymphalini & 5 & 5 & 0 & 5 & 5 & 0 & 0 & 100 \\
\hline Kallimini & 5 & 5 & 0 & 5 & 4 & 1 & 0 & 88,9 \\
\hline Melitaeini & 9 & 9 & 0 & 9 & 9 & 0 & 0 & 100 \\
\hline Limenitidinae & 15 & 15 & $\mathbf{0}$ & 9 & 10 & 5 & 6 & 42,1 \\
\hline
\end{tabular}


Tabela 2. Continuação...

\begin{tabular}{|c|c|c|c|c|c|c|c|c|}
\hline \multirow[t]{3}{*}{ Taxa } & \multicolumn{2}{|c|}{ Número } & \multirow[t]{3}{*}{$\begin{array}{c}\text { Espécies de Campo } \\
\text { Natural }\end{array}$} & \multicolumn{2}{|c|}{ Amostragens } & \multicolumn{2}{|c|}{$\begin{array}{l}\text { Espécies } \\
\text { exclusivas }\end{array}$} & \multirow[t]{3}{*}{ Is } \\
\hline & Sp. & Spp. & & 1944 & 2005 & 1944 & 2005 & \\
\hline & & & & 2002 & 2010 & 2002 & 2010 & \\
\hline Heliconiinae & 22 & 22 & $\mathbf{0}$ & 20 & 21 & 1 & 2 & 92,7 \\
\hline Argynnini & 2 & 2 & 0 & 2 & 1 & 1 & 0 & 66,7 \\
\hline Acraeini & 9 & 9 & 0 & 7 & 9 & 0 & 2 & 87,5 \\
\hline Heliconiini & 11 & 11 & 0 & 11 & 11 & 0 & 0 & 100 \\
\hline Total & 689 & 692 & 42 & 572 & 543 & 148 & 117 & 76 \\
\hline
\end{tabular}

Papilionidae, compostas de espécies conspícuas e mais facilmente registráveis (Brown Jr. 1992, Brown Jr. \& Freitas 1999).

No início das coletas, os Campos Naturais estiveram presentes por um longo período (Maack 1968), até meados de 1970, quando os remanescentes foram crescentemente substituídos por monoculturas. Os registros atuais para as espécies de Campo Natural se referem a três pequenas manchas menores que cinco hectares, duas das quais extintas. A lepidopterofauna desta fitofisionomia está criticamente sob ameaça, pois nenhuma unidade de conservação abriga tal vegetação. Há inclusive espécies da lista vermelha da fauna ameaçada do Paraná que são endêmicas dessa vegetação, e apenas E. corethrus foi reencontrada, porém, a pequena área onde a população dessa espécie habitava, foi substituída por pastagem para gado, extinguindo a única população atualmente conhecida no estado (Dolibaina et al 2010).

Assim como Mielke et al. (2008) proporam dados de Hesperiidae endêmicos do Cerrado, indicamos aqui as espécies de Hesperioidea e Papilionoidea características de Campo Natural, com o intuito de subsidiar medidas conservacionistas, principalmente no estabelecimento de novas unidades de conservação permanentes que protejam essa vegetação característica, e por consequência sua fauna.

No trabalho de Tyler et al. (1994) há dados indicando a ocorrência conjunta entre subespécies de M. lysythous no sul do Brasil, já para $P$. bunichus, enquanto $P$. b. bunichus voa acima de $800 \mathrm{~m}$ de altitude, $P$. b. perrhebus foi apenas encontrada nas altitudes mais baixas da região (vale do rio Ivaí). Quanto a $E$. socus, já eram conhecidas outras regiões como Joinville e São Bento do Sul em Santa Catarina e Foz do Iguaçu e Londrina no Paraná (DZUP), onde as subespécies socus e pseudexadeus voam juntas. Estudos taxonômicos são necessários para tais taxa.

\section{Agradecimentos}

Agradecemos a Anderson Luis Tosetto da RPPN Paraná pelo apoio logisítico em algumas coletas. Aos amigos Vitor Hugo Gonçalves e Edivando Alves pelo auxílio nos trabalhos de campo. Ao Sr. Márcio Canto de Miranda, proprietário da RPPN Ninho do Corvo, pela disponibilidade da área para estudo. A Fábio Dangui por disponibilizar a coleção particular de Hipólito Schneider e Cristiane Hiert a do Museu de História Natural de Guarapuava. Agradecemos também a Alfred Moser pela identificação dos Lycaenidae, a Eduardo Carneiro e a dois revisores anônimos por valiosas sugestões ao trabalho e Fernando Maia Silva Dias pelo Abstract. Ao CNPq pela concessão das bolsas de produtividade e mestrado aos autores.

\section{Referências Bibliográficas}

BIEZANKO, C.M. 1938a. Sobre alguns lepidópteros que ocorrem em arredores de Curitiba (Estado do Paraná). Apontamentos lepidopterológicos feitos em 1932. Livraria Globo, Pelotas.
BIEZANKO, C.M. 1938b. Dois meses de caça lepidopterológica nos arredores do Porto União e União da Vitória, em outubro e novembro de 1932. Contribuição ao conhecimento da fauna dos insetos de Santa Catarina e Paraná. R. de Agron. 2(16-17):1-11.

BIEZANKO, C.M. 1960. Satyridae, Morphidae et Brassolidae da zona sueste do Rio Grande do Sul. Arq. Ent. série A 4:1-12.

BIEZANKO, C.M. 1963. Hesperiidae da zona sueste do Rio Grande do Sul. Arq. Ent. série A 6:1-24.

BIEZANKO, C.M. \& FREITAS, R. G. 1938. Catálogo dos insetos encontrados na cidade de Pelotas e arredores. Fasciculo 1. Lepidopteros. Bol. Esc. Agr. Eliseu Maciel. 25:1-32.

BIEZANKO, C.M. \& MIELKE, O.H.H. 1973. Contribuição ao estudo faunístico dos Hesperiidae americanos. IV. Espécies do Rio Grande do Sul, Brasil, com notas taxonômicas e descrições de espécies novas. (Lepidoptera). Acta Biol. Parana. 2(1-4):51-102.

BIEZANKO, C.M., MIELKE, O.H.H. \& WEDDERHOFF, A. 1978. Contribuição ao estudo faunístico dos Riodinidae do Rio Grande do Sul, Brasil (Lepidoptera). Acta Biol. Parana. 7(1-4):7-22.

BIEZANKO, C.M. \& PITOÑ, J. 1941. Breves apontamentos sobre alguns lepidopteros encontrados nos arredores de Itaiópolis. Bol. Esc. Agr. Eliseu Maciel. 28:1-24.

BONFANTTI, D., DI-MARE, R.A. \& GIOVENARDI, R. 2009. Butterflies (Lepidoptera: Papilionoidea and Hesperioidea) from two forest fragments in northern Rio Grande do Sul, Brazil. Check List. 5(4):819-829. http:// www.checklist.org.br/getpdf?SL035-09 (último acesso em 25/03/2010).

BROWN Jr., K.S. 1992. Borboletas da Serra do Japi: diversidade, habitats, recursos alimentares e variação temporal. In História natural da Serra do Japi: ecologia e preservação de uma área florestal no sudeste do Brasil (L.P.C. MORELLATO, ed.). Unicamp; FAPESP, Campinas, p.142-187.

BROWN Jr., K.S. \& FREITAS, A.V.L. 1999. Lepidoptera. In Biodiversidade do Estado de São Paulo: síntese do conhecimento ao final do século XX (JOLY, C.A. \& BICUDO C.E.M., Org). Invertebrados Terrestres (C.R.F. BRANDÃO \& E.M. CANCELLO, eds.). São Paulo: FAPESP. v. 5, p.226-243.

BROWN Jr., K.S. \& FREITAS, A.V.L. 2000. Atlantic forest butterflies: indicators for landscape conservation. Biotropica 32(4b):934-956.

BUSTOS, E.O.N. 2009. Mariposas diurnas (Lepidoptera: Papilionoidea y Hesperioidea) del Parque Nacional Iguazú, Provincia de Misiones, Argentina. Trop. Lepid. Res. 19(2):71-81.

CARNEIRO, E., MIELKE, O.H.H. \& CASAGRANDE, M.M. 2008a. Borboletas do sul da ilha de Santa Catarina, Florianópolis, Santa Catarina, Brasil (Lepidoptera: Hesperioidea e Papilionoidea). SHILAP Revta. Lepid. 36(142):261-271.

CARNEIRO, E., MIELKE, O.H.H. \& CASAGRANDE, M.M. 2008b. Inventário de borboletas no Brasil: estado da arte e modelo de áreas prioritárias para pesquisa com vistas à conservação. Nat. \& Conserv. 6(2): 68-90: http://internet.boticario.com.br/Internet/staticFiles/Fundacao/pdf/ ARTIGOS_NC12/11N\&C_V6_N2_SANTOS_PORT.pdf. 
CASAGRANDE, M.M. \& MIELKE, O.H.H. 2008. Lepidoptera: Charonias theano theano (Boisduval, 1836). In Livro Vermelho da fauna ameaçada de extinção. (A.B.M. Machado, G.M. Drummond, \& A.P. Paglia, orgs.). Biodiversidade 19, Brasília, v. 1, cap. 46, p.438-439.

CORSEUIL, E., QUADROS, F.C., TESTON, J.A. \& MOSER, A. 2004. Borboletas (Lepidoptera, Papilionoidea e Hesperioidea) ocorrentes no Centro de Pesquisas e Conservação da Natureza Pró-Mata. 4. Lycaenidae. Comun. Mus. Cienc. Tecnol. PUCRS, Sér. zool. 9: 65-70.

DOLIBAINA, D.R., CARNEIRO, E., DIAS, F.M., MIELKE, O.H.H. \& CASAGRANDE, M.M. 2010. Registros inéditos de borboletas (Papilionoidea e Hesperioidea) ameaçadas de extinção para o Estado do Paraná, Brasil: novos subsídios para reavaliação dos critérios de ameaça. Biota Neotrop. 10 (3): http://www.biotaneotropica.org.br/v10n3/pt/ abstract?article+bn01210032010 (último acesso em 26/10/2010).

EBERT, H. 1969. On the frequency of butterflies in eastern Brazil, with a list of butterfly fauna of Poços de Caldas, Minas Gerais. J. Lepid. Soc. 23 supl.3: 1-48.

EMERY, E.O., BROWN Jr., K.S. \& PINHEIRO, C.E.G. 2006. As borboletas (Lepidoptera, Papilionoidea) do Distrito Federal, Brasil. Rev. Bras. Ent. 50(1):85-92.

GIOVENARDI, R., DI-MARE, R.A., SPONCHIADO, J., ROANI, S.H., JACOMASSA, F.A.F., JUNG, A.B. \& PORN, M.A. 2008. Diversidade de Lepidoptera (Papilionoidea e Hesperioidea) em dois fragmentos de floresta no município de Frederico Westphalen, Rio Grande do Sul, Brasil. Rev. Bras. Ent. 52(4):599-605.

INSTITUTO AGRONÔMICO DO PARANÁ - IAPAR. 2010. Cartas climáticas - médias históricas. http://www.iapar.br/arquivos/Image/ monitoramento/Medias_Historicas/Guarapuava.htm (último acesso em 25/03/2010).

ISERHARD, C.A., QUADROS, M.T., ROMANOWSKI, H.P. \& MENDONÇA Jr., M.S. 2010. Borboletas (Lepidoptera: Papilionoidea e Hesperioidea) ocorrentes em diferentes ambientes na Floresta Ombrófila Mista e nos Campos de Cima da Serra do Rio Grande do Sul, Brasil. Biota Neotrop. 10(1): http://www.biotaneotropica.org.br/v10n1/pt/ abstract?article+bn02910012010 (último acesso em 26/10/2010).

LAMAS, G.M. (ed.) 2004. Checklist: part 4A Hesperioidea - Papilionoidea p. XXXV + 439. In Atlas of Neotropical Lepidoptera (J.B HEPPNER, ed.). Association of Tropical Lepidoptera, Gainesville, v.5A.

LINK, D., BIEZANKO, C.M., TARRAGO, M.F. \& CARVALHO, S. 1977. Lepidoptera de Santa Maria e arredores. I Papilionidae e Pieridae. Rev. Cent. Cienc. Rurais, Univ. Fed. Santa Maria 7(4):381-389.

MAACK, R. 1968. Geografia Física do Estado do Paraná. Universidade Federal do Paraná; Instituto de Biologia e Pesquisa Tecnológica, Curitiba, p.350.

MIELKE, C.G.C. 1995. Papilionoidea e Hesperioidea (Lepidoptera) de Curitiba e seus arredores, Paraná, Brasil, com notas taxonômicas sobre Hesperiidae. Rev. Bras. Zool. 11(4):759-776.

MIELKE, O.H.H. 1968. Contribuição ao estudo faunístico dos "Hesperiidae" brasileiros I. Resultados de uma excursão a Foz do Iguaçu, Paraná, Brasil, com notas taxonômicas (Lepidoptera). Atas Soc. Biol. Rio J. 12(2):73-78.
MIELKE, O.H.H. \& CASAGRANDE, M.M. 1998. Papilionoidea e Hesperioidea (Lepidoptera) do Parque Estadual do Morro do Diabo, Teodoro Sampaio, São Paulo. Brasil. Rev. Bras. Zool. 14(4):967-1001.

MIELKE, O.H.H. \& CASAGRANDE, M.M. 2004. Borboletas. In Livro vermelho da fauna ameaçada no Estado do Paraná (S.B. Mikich \& R.S. Bernils, orgs.). Instituto Ambiental do Paraná, Curitiba, p. 713-739

MIELKE, O.H.H., EMERY, E.O. \& PINHEIRO, C.E.G. 2008. As borboletas Hesperiidae (Lepidoptera, Hesperioidea) do Distrito Federal, Brasil. Rev. Bras. Ent. 52(2):283-288.

PAZ, A.L.G., ROMANOWSKI, H.P. \& MORAIS, A.B.B. 2008. Nymphalidae, Papilionidae e Pieridae (Lepidoptera: Papilionoidea) da Serra do Sudeste do Rio Grande do Sul, Brasil. Biota Neotrop. 8(1): http://www. biotaneotropica.org.br/v8n1/pt/abstract?inventory+bn01608012008 (último acesso em 15/02/2009).

PINHEIRO, C.E.G. \& EMERY, E.O. 2006. As borboletas (Lepidoptera: Papilionoidea \& Hesperioidea) da Área de Proteção Ambiental do Gama e Cabeça de Veado (Distrito Federal, Brasil). Biota Neotrop. 6(3): http:// www.biotaneotropica.org.br/v6n3/pt/fullpaper?bn01506032006+pt (último acesso em 20/03/2010).

RIBEIRO, M.C., METZGER, J.P., MARTENSEN, A.C., PONZONI, F.J. \& HIROTA, M.M. 2009. The Brazilian Atlantic Forest: how much is left, and how is the remaining forest distributed?: implications for conservation. Biol. Conserv. 142:1144-1156.

SACKIS, G.D. \& MORAIS, A.B.B. 2008. Borboletas (Lepidoptera: Hesperioidea e Papilionoidea) do campus da Universidade Federal de Santa Maria, Santa Maria, Rio Grande do Sul. Biota Neotrop. 8(1): http:// www.biotaneotropica.org.br/v8n1/pt/abstract?inventory+bn01908012008 (último acesso em 25/03/2010).

SOCIEDADE DE PROTEÇÃO DA VIDA SELVAGEM - SPVS. 1996. Nossas árvores - manual para a recuperação da reserva florestal legal. FNMA, Curitiba.

SUMMERVILLE, K. BOULWARE, M.J., VEECH, J.A. \& CRIST, T.O. 2003. Spatial variation in species diversity and composition of forest Lepidoptera in Eastern Deciduous Forests of North America. Cons. Biol. 17(4): 1045-1057.

TESTON, J.A. \& CORSEUIL, E. 1999. Borboletas (Lepidoptera, Rhopalocera) ocorrentes no Centro de Pesquisa e Conservação da Natureza Pró-Mata. 1. Papilionidae. Biociências 4(2):217-228.

TESTON, J.A. \& CORSEUIL, E. 2000. Borboletas (Lepidoptera, Rhopalocera) ocorrentes no Centro de Pesquisa e Conservação da Natureza Pró-Mata. 2. Pieridae. Biociências 5:143-155.

TESTON, J.A. \& CORSEUIL, E. 2002. Borboletas (Lepidoptera, Rhopalocera) ocorrentes no Centro de Pesquisa e Conservação da Natureza Pró-Mata. 3. Nymphalidae. Comun. Mus. Cienc. Tecnol. PUCRS 7:143-155.

TYLER, H., BROWN Jr., K.S. \& WILSON, K. 1994. Swallowtail butterflies of the Americas: a study in biological dynamics, ecological diversity, biosystematics, and conservation. Scientific Publishers, Gainesville, 377p.

UEHARA-PRADO, M., BROWN Jr., K.S. \& FREITAS, A.V.L. 2007. Species richness, composition and abundance of fruit-feeding butterflies in the Brazilian Atlantic Forest: comparison between a fragmented and a continuous landscape. Global Ecol. Biogeogr. 16:43-45. 